ISSN 0819-2642

ISBN 0734025602

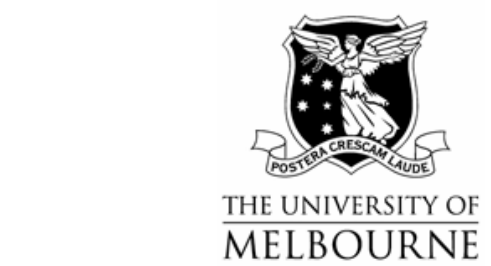

THE UNIVERSITY OF MELBOURNE

DEPARTMENT OF ECONOMICS

RESEARCH PAPER NUMBER 904

MAY 2004

\title{
VERTICAL INTEGRATION IN THE PRESENCE OF UPSTREAM COMPETITION
}

by

Catherine C. de Fontenay

$\&$

Joshua S. Gans

Department of Economics

The University of Melbourne

Melbourne Victoria 3010

Australia. 


\section{Vertical Integration in the Presence of Upstream Competition}

by

Catherine C. de Fontenay and Joshua S. Gans University of Melbourne

First Draft: $5^{\text {th }}$ April, 2002

This Version: $25^{\text {th }}$ August, 2003

We analyze vertical integration in the case of upstream competition and compare outcomes to the case where upstream assets are owned by a single agent (i.e., upstream monopoly). In so doing, we make two contributions to the modelling of strategic vertical integration. First, we base industry structure namely, the ownership of assets - firmly within the property rights approach to firm boundaries. Second, we model the potential multilateral negotiations using a fully specified, non-cooperative bargaining model designed to easily compare outcomes achieved under upstream competition and monopoly. Given this, we demonstrate that vertical integration can alter the joint payoff of integrating parties in ex post bargaining; however, this bargaining effect is stronger for firms integrating under upstream competition than upstream monopoly. We also integrating under upstream competition than upstream monopoly. We also
consider the potential for integration to internalize competitive externalities in a consider the potential for integration to internalize competitive externalities in a
manner that cannot be achieved under non-integration; i.e., by favouring internal manner that cannot be achieved under non-integration; i.e., by favouring internal over external supply. We demonstrate that ex post monopolization is more likely
to occur when there is an upstream monopoly than when there is upstream competition. Our general conclusion is that the simple intuition that the presence of upstream competition can mitigate and reduce the incentives for socially undesirable vertical integration is misplaced and, depending upon the strength undesirable vertical in downstream competition (i.e., product differentiation), the opposite could easily

Keywords. vertical integration, foreclosure, monopolization, bargaining, competition.

Melbourne Business School and Department of Economics, University of Melbourne. E-mail al comments to JGans@unimelb eduau. The authors would like to thank Stephen King Jeff Zwiebel, two anonymous referees and seminar participants at the IDEI/University of Toulouse, University of Texa (Austin) and Northwestern University for helpful comments. Responsibility for all errors remains our own. The latest version of this paper is available at http://www.mbs.edu/gans. 


\section{Introduction}

There are long-standing antitrust concerns about potential social detriment from vertical integration, centering on integration by an upstream monopoly into a downstream segment. The monopolist may restrict supply after integration, foreclose on downstream rivals, or it may appropriate more rents at the expense of downstream firms. Moreover, there is a general belief that improving competition in the bottleneck segment would alleviate these concerns. ${ }^{1}$

There are two ways that competition might serve to discourage socially harmful vertical integration. First, upstream competitors will respond to attempts by a firm to foreclose on non-integrated downstream firms by expanding their supply to them. This undermines the ability of an upstream firm to use vertical integration to raise prices in the industry by restricting supply to some downstream firms. Second, it is claimed that competition reduces any bargaining power conferred on the monopolist by integration and any foreclosure threats.

To date, there has been no unified theoretical analysis of the role that competition plays on the incentives for vertical integration and its social desirability. This paper provides such an analysis. In so doing, our primary task is to provide a model capable of studying the pure effect of an increase in competition. Thus, we need to consider an environment where competition does not otherwise change total resources, technical productivity or the nature of bargaining in the industry in an ad hoc way. To this end, we consider an environment where there are two downstream and two upstream assets. Upstream competition is modeled as a situation where the two upstream assets are separately owned, whereas under upstream monopoly they are commonly owned.

Our main modeling contribution, however, lies in the game we use to model bargaining between upstream and downstream firms over input supply. We consider an environment, common in the property rights approach to firm boundaries (Grossman and

${ }^{1}$ See Williamson (1987) for a discussion of these presumptions. The US Department of Justice merge guidelines (DOJ, 1984) state that vertical mergers are only likely to attract concern if concentration in a market (in practice, an upstream market) is high. 
Hart, 1986; Hart and Moore, 1990), where the manager of each asset has asset-specific skills, and integration decisions - i.e., the ownership of assets - are made prior to bargaining over the supply of inputs. This set-up allows us to consider the bargaining effects of vertical integration in a similar manner to the standard property rights literature. Importantly, in our environment, integration does not remove the potential for the manager of an acquired firm to earn rents. This is true both for a firm integrating vertically but also for an upstream monopoly where one upstream asset is owned by the manager of the other. Thus, we can capture the full effects of integration on bargaining relations in the industry. Moreover, in so doing, we are able to investigate new issues in strategic vertical integration; namely, the potential differences between forward and backwards integration. ${ }^{2}$

Bargaining takes a non-cooperative form with each upstream-downstream pair negotiating sequentially over the quantity supplied and a price between them. A key feature of our bargaining game is that changes in market structure can change supply arrangements, either because such arrangements can be renegotiated (as in Stole and Zwiebel, 1996) or because they are made contingent on changes in market structure (as in Inderst and Wey, 2003). We demonstrate that this type of bargaining leads naturally to some of the inefficiencies emphasised in the contracting externalities literature: an upstream supplier with more than one buyer downstream oversupplies the market, because they cannot commit not to impose negative externalities on one buyer by selling large quantities to the other buyer. ${ }^{3}$ Nonetheless, we are able to characterise surplus division; relating the realised payoffs of upstream and downstream firms to their relative power if sets of supply relationships were to be severed. Indeed, the payoffs resemble a 'coalition structure' similar to those derived in cooperative game theory, albeit over a

${ }^{2}$ Most analyses of the competitive impacts of vertical integration make no distinction between the type of integration (e.g. Riordan and Salop, 1995; Klass and Salinger, 1995; and Hovenkamp, 2001). The reason for this is that both parties have to agree to merge and so it is generally held to be in their joint interest. for this is that both parties have to agree to merge and so it is generally held to be in their joint interest. However, when there are many firms, as is well known, changes in asset ownership have differential
impacts on different types of agents (Hart and Moore, 1990). We demonstrate that this is the case for impacts on different types of agents (Hart and Moore, 1990). We demonstrate that this is the case for
vertical integration in general as forward and backward integration have different returns to the merging parties and different effects on outsiders. While the distinction is overlooked in competition policy, our analysis highlights where it may be important and given more credence.

3 The seminal work on this comes from Hart and Tirole (1990) in terms of its relationship to vertical integration. However McAfe and Schwartz (1994), O'Brien and Shafer (1992) and Segal (1999) provide comprehensive treatments of the contracting problem when there are externalities amogst firms. See Rey 
reduced industry surplus.

We demonstrate that vertical integration has two potential effects. First, the bargaining position of all agents changes. Second, some contracting externalities are internalised. To demonstrate the first, we initially consider an environment where downstream assets are in different markets so that there are no competitive externalities between them (Section 3). There vertical integration changes only the distribution of bargaining power and not the surplus generated. We show that vertical integration can increase the sum of payoffs for the integrating parties because it improves their bargaining position in negotiations with independent firms; specifically, it eliminates the possibility of market structures that may be favourable to independents.

Importantly, we demonstrate that there is a greater incentive for vertical integration under upstream competition than under monopoly. This is because the bargaining benefits come from the redistribution of rents from non-integrating parties; and in a monopoly, the non-integrating parties already have low rents. Thus, competition enhances rather than reduces the potential for purely strategic vertical integration. Moreover, we find that integration occurs from the more competitive segment into the less competitive segment: for example, forward integration is chosen over backward integration only when upstream firms are closer substitutes (in terms of generating overall industry profits) than downstream firms.

When competitive externalities downstream are taken into account, there is an additional incentive for vertical integration: integration can internalise those externalities and lead to some degree of monopolization in the industry. The integrated upstream firm, when dealing with the non-integrated downstream firm, will internalise the effect of its supply on its own downstream firm. Vertical integration of an upstream monopolist leads to higher industry profits than are possible under upstream competition, raising the returns to integration under upstream monopoly relative to upstream competition and mitigating the returns identified earlier that were based purely on bargaining. Indeed, we demonstrate that, in some situations, industry profits may fall (along with consumer surplus) as a result of vertical integration under upstream competition.

In this environment, we identify product differentiation as a key parameter driving

and Tirole (2003) for a survey. 
incentives to vertically integrate. In particular, we find that when product differentiation is low (high), backward integration is more (less) privately profitable than forward integration. Importantly, while the conventional concern about vertical integration is confirmed when downstream products are relatively homogeneous, the incentive for such integration will be higher under upstream competition than upstream monopoly if products are relatively differentiated. Both these results suggest that the conventional approach of examining the market power of the acquiring firm will not necessarily allow one to draw a conclusion as to whether vertical integration is anti-competitive or not.

The paper that is closest to our own is that of Hart and Tirole (1990) - hereafter, HT. That paper is the first to identify the bargaining and monopolisation effects that arise from vertical integration. ${ }^{4}$ While their paper identifies these using three separate variants - each with extreme assumptions regarding downstream demand and upstream costs our model nests all of those variants within a single model that allows for more general downstream and upstream environments; in particular, we allow for downstream product differentiation that is identified as an important driver of incentives for integration. ${ }^{5}$ Thus, one contribution of our paper is to demonstrate the robustness of HT's results. ${ }^{6}$ Nonetheless, we identify subtle differences between our conclusions and theirs throughout. For instance, as in HT, we demonstrate that in some cases vertical integration may lead to a situation where there is foreclosure in input supply to the non-integrated downstream firm. However, in our model, this does not necessarily imply there is

${ }^{4}$ Bolton and Whinston (1993) also identify a bargaining effect from vertical integration. Their model, however, does not have downstream firms directly competing, focusing instead of the impact of bargaining on investment incentives. Their analysis is complementary with that here although, like HT, it is formulated in a special manner to remove any distinction between forward and backward integration.

${ }^{5}$ A recent paper by Chemla (2003) also nests a bargaining and monopolization effect. He demonstrates that an upstream monopolist may expend resources to encourage entry by downstream firms so as to limit their bargaining power. He demonstrates that vertical integration will have the dual effect of reducing the monopolist's need to expend those resources and also lead to higher industry profits. de Fontenay and Gans monopolist's need to expend those resources and also lead to higher industry profits. de Fontenay and Gans
(1999) similarly demonstrate that vertical integration can lead to reduced downstream entry and higher industry profits, but do so using a bargaining framework similar to that considered in this paper, although industry profits, but do so using a bargaining framework similar to that considered in this paper, although
without an incomplete-contracts perspective on the effect of integration. The current paper does not study the effect of changes in bargaining power the entry decisions of firms, but focuses its attention on the effect of upstream competition. ${ }^{6}$ Klass and Salinger (1995) argued that HT's results were highly specific and may not carry over to more
general environments. Indeed, as they note, many of HT's results rely on integration precipitating exit of an upstream or downstream firm. We demonstrate similar bargaining and monopolization effects to HT but without the use of the exit device that drove many of their results (in addition, to our more general technology and demand assumptions). 
foreclosure in payments to that firm, as the integrated firm is interested in preserving the option to supply to that firm if bargaining with its internal manager were to break down.

Significantly, however, HT's model is not equipped to properly examine the questions that motivate us here. First, they assume that upstream and downstream firms simply share the surplus arising from a negotiation according to a fixed parameter, rather than model the drivers of bargaining power-in particular, the asset-specific skills that confer bargaining power in the property rights literature. ${ }^{7}$ Consequently, there is no distinction between forward and backward integration. In contrast, in our model, the bargaining position of each firm is driven by their roles in possible market structures that arise following breakdowns in individual negotiations. As forward and backwards integration have different implications as to what market structures are feasible, there will be a difference in the incentives and impact of each.

Second, their analysis of the impact of upstream competition is limited to an analysis of the efficiency of the weaker upstream firm. That is, they consider what happens to the incentives to vertically integrate as the weaker upstream firm becomes more efficient, which confounds the effect of market power and the effect of superior productivity. Our analysis of the impact of upstream competition models monopoly as the horizontal integration of both upstream assets. And as such, it explicitly considers the impact of vertical integration on internal arrangements within the upstream monopoly.

In terms of its bargaining game, the paper has several antecedents. Grossman and Hart (1986) and Hart and Moore (1990) were the first to focus on Shapley values as likely outcomes of the bargaining game between firms. Variants of the bargaining game developed by Stole and Zwiebel (1996) have been applied to bargaining between firms over variable quantities by de Fontenay and Gans (1999, 2003b), Inderst and Wey (2003) and Björnerstedt and Stennek (2001). ${ }^{8}$ Note that contracting externalities are ruled out in all of the above game structures: considering environments in which downstream players impose no externalities on each other. Here instead we allow sequential contracting in an environment in which downstream players are in the same market, leading to contract

${ }^{7}$ Other papers in the literature avoid the need to model the drivers of bargaining power by assuming that either upstream or downstream firms have all of the bargaining power (Rey and Tirole, 2003; Chemla,
2003 ). This is also a common assumption in the contracting with externalities literature (McAfee and Schwartz, 1994; and Segal, 1999). 
externalities as explored elsewhere in the literature on vertical integration.

The remainder of the paper proceeds as follows: Section 2 sets up our basic model and, in particular, the non-cooperative bargaining game that is capable of assessing the impact of upstream competition on the incentives for vertical integration. Sections 3 and 4 then provide analyses of the 'no externalities' and 'competitive externalities' cases when one vertical merger is possible. Section 5 considers incentives for a counter merger and the question of whether the possibility of such mergers may alter incentives for the initial merger. A final section concludes.

\section{$2 \quad$ Model Set-Up}

We examine an industry that has two upstream and two downstream assets. The upstream assets produce inputs that are used by downstream assets to make final goods. Inputs from at least one upstream asset are necessary for valuable production downstream. In addition, associated with each asset is a manager endowed with assetspecific human capital that is in turn necessary to generate valuable goods and services from that asset. ${ }^{9}$ We denote the respective managers of upstream firms $A$ and $B$ by $U_{A}$ and $U_{B}$, and downstream managers by $D_{1}$ and $D_{2}$. Integration changes the ownership of these assets; however, the manager associated with an asset will not change, as each remains necessary for its use.

An upstream asset, $U_{j}$, can produce input quantities $q_{1 j}$ and $q_{2 j}$ for $D_{1}$ and $D_{2}$, respectively. Its costs are given by $c_{j}\left(q_{1 j}, q_{2 j}\right)$, assumed to be weakly convex in $\left(q_{1 j}, q_{2 j}\right)$

Using input quantities, $q_{i A}$ and $q_{i B}$ from $U_{A}$ and $U_{B}$, respectively, $D_{i}$ makes a downstream profit (gross of payments to upstream suppliers) of $\pi_{i}\left(q_{i A}, q_{i B}, q_{-i A}, q_{-i B}\right)$ where $-i$ denotes the index of $i$ 's potential downstream rival. We assume that $\pi_{i}($.$) is$ concave in $\left(q_{i A}, q_{i B}\right)$, non-increasing in $\left(q_{-i A}, q_{-i B}\right)$.

${ }^{8}$ Only de Fontenay and Gans examine vertical integration; Inderst and Wey examine horizontal mergers.

${ }_{9}^{9}$ This is a common set-up in the incomplete contracts literature (see, for example, Bolton and Whinston, 
Finally, it will often be convenient to express outcomes in terms of industry profits that can be generated for alternative configurations of supply relationships. Let $\Pi\left(\overline{D_{1} D_{2} U_{A} U_{B}}\right) \equiv \max _{\substack{q_{1, A}, q_{1 B} \\ q_{2 A}, q_{2 B}}} \pi_{1}\left(q_{1 A}, q_{1 B}, q_{2 A}, q_{2 B}\right)+\pi_{2}\left(q_{2 A}, q_{2 B}, q_{1 A}, q_{1 B}\right)-c_{A}\left(q_{1 A}, q_{2 A}\right)-c_{B}\left(q_{1 B}, q_{2 B}\right)$

be maximized industry profits when both upstream assets can potentially provide inputs that can be used by both downstream assets. Industry profits for other supply possibilities are similarly defined. For example,

$$
\begin{gathered}
\Pi\left(\overline{D_{1} U_{A} U_{B}}\right) \equiv \max _{q_{1 A}, q_{1 B}} \pi_{1}\left(q_{1 A}, q_{1 B}, 0,0\right)-c_{A}\left(q_{1 A}, 0\right)-c_{B}\left(q_{1 B}, 0\right) \\
\Pi\left(\overline{D_{1} U_{A}}\right) \equiv \max _{q_{1 A}} \pi_{1}\left(q_{1 A}, 0,0,0\right)-c_{A}\left(q_{1 A}, 0\right)
\end{gathered}
$$

The Le Châtelier principle implies that maximised industry profits are higher whenever an additional asset and its associated manager are used. For example, $\Pi\left(\overline{D_{1} D_{2} U_{A}}\right) \geq \Pi\left(\overline{D_{2} U_{A}}\right)$ and $\Pi\left(\overline{D_{1} D_{2} U_{A} U_{B}}\right) \geq \Pi\left(\overline{D_{1} D_{2} U_{A}}\right)$.

It is possible that a particular market structure may involve a 'partitioned' set of supply arrangements. For instance, $D_{1}$ may only negotiate with $U_{A}$ and $D_{2}$ may only negotiate with $U_{B}$. For this situation, let $\left(\hat{q}_{1 A}, \hat{q}_{2 B}\right)$ be the equilibrium input supply quantities. ${ }^{10}$ Then,

$$
\begin{aligned}
& \Pi\left(\overline{D_{1} U_{A}}, \overline{D_{2} U_{B}}\right) \equiv \pi_{1}\left(\hat{q}_{1 A}, 0,0, \hat{q}_{2 B}\right)-c_{A}\left(\hat{q}_{1 A}, 0\right) \\
& \Pi\left(\overline{D_{2} U_{B}}, \overline{D_{1} U_{A}}\right) \equiv \pi_{2}\left(0, \hat{q}_{2 B}, \hat{q}_{1 A}, 0\right)-c_{B}\left(0, \hat{q}_{2 B}\right)
\end{aligned}
$$

denotes the (equilibrium) profits to each buyer/supplier pair, respectively.

\section{$2.1 \quad$ Timeline}

The timeline for our model is as follows

STAGE 0 (Asset Allocation): Ownership of assets is determined among all four managers.

STAGE 1 (Bargaining): Bargaining over input supply terms takes place. STAGE 2 (Production): Production takes place and payoffs are realised.

Initially, the asset allocation process is not modeled as a fully specified endogenous

1993 and Hart, 1995).
$\begin{aligned} & \text { Below we demonstrate that this equilibrium is Cournot, i.e., that } \\ & \hat{q}_{1 A}=\arg _{\max }, \pi_{1}\left(q_{1 A}, 0,0, \hat{q}_{2 B}\right)-c_{A}\left(q_{1 A}, 0\right) \text { and } \hat{q}_{2 B}=\arg \max _{q_{2 B}} \pi_{2}\left(0, q_{2 B}, \hat{q}_{1 A}, 0\right)-c_{B}\left(0, q_{2 B}\right) \text {. In actuality, }\end{aligned}$


process. That is, we focus on more limited, partial incentives, including whether integration is jointly profitable for the merging parties. Nonetheless, in Section 5, we consider the possibility of counter-mergers and possible equilibria in Stage 0 to check (and, in general, confirm) the robustness of this partial approach. For now, the stage that requires further elaboration is the bargaining stage and we turn now to discuss that in detail.

Note that we do not explicitly model any efficiency cost to integration. This could involve a straight resource costs (as in HT) or alternatively investment incentive effects (as in Hart and Moore, 1990; Bolton and Whinston, 1993). It would be straightforward to incorporate both upstream and downstream investment into the model here, however, they are omitted so as to focus on the main effects as they relate to competition. Essentially, the impact of integration on such investment will involve a similar set of effects as those considered by Segal and Whinston (2000) for the exclusive dealing case. For the remainder of this paper, we simply compare the profitability of integration under different market structures, supposing that the most profitable opportunities of integration are the least likely to be outweighed by the cost of lost resources or investment.

\section{$2.2 \quad$ Bargaining}

Bargaining is bilateral, vertical (occurring between managers of individual upstream and downstream assets), and sequential (only one pair of agents bargain at a time). Each upstream-downstream pair negotiates over price and quantity supply terms. For example, $U_{j}$ and $D_{i}$ bargain over terms specifying a quantity of inputs purchased, $q_{i j}$, and a lump-sum transfer, $\tilde{p}_{i j}$ paid by $i$ to $j$. When bargaining takes place internally, quantity is not relevant and the focus of negotiations is over the size of any transfer payment, $\tilde{i}_{i j}$ paid by $j$ to manager $i$ for $i$ 's participation in the production process.

Our bargaining game takes a particular extensive form. ${ }^{11}$ The game is as follows: 
fix an order of pairs to negotiate in sequence. This order is common knowledge and, as will be demonstrated, irrelevant for the equilibrium outcome. Each pair negotiates bilaterally in a manner specified by Binmore, Rubinstein and Wolinsky (1986); i.e., each makes sequential offers to one other until they reach an agreement, and after an offer is rejected there is an infinitesimal probability of an irrevocable breakdown in their negotiations. Once an agreement is reached, the next pair begins bargaining. ${ }^{12}$ If a breakdown occurs before an agreement is reached, the entire sequence of negotiations takes place again (in the same order as before), but without any pair whose negotiations have broken down previously. Once all pairs have either agreed or suffered a breakdown, the game ends.

Figure 1 presents a possible sequence of bargaining negotiations for the baseline case of non-integration. Each box represents a bargaining session between a pair which can result in agreement (A) or breakdown (B). Г(.) denotes the subgame which takes place over the indicated sequence of pairs. Thus, $\Gamma\left(D_{1} U_{A}, D_{2} U_{B}, D_{1} U_{B}, D_{2} U_{A}\right)$ indicates a sequence of negotiations beginning with $D_{1}-U_{A}$, followed by $D_{2}-U_{B}, D_{1}-U_{B}$ and $D_{2}-U_{A}$, respectively. If there is a breakdown in negotiations between $D_{1}$ and $U_{A}$ in this sequence, the renegotiation subgame, $\Gamma\left(D_{2} U_{B}, D_{1} U_{B}, D_{2} U_{A}\right)$, is triggered. Thus, breakdowns trigger a sequence of renegotiations between all remaining pairs in the original order. ${ }^{13}$ Consequently, when agents bargain together, they take as their disagreement payoff their payoff from this renegotiation game.

There are two key assumptions of this bargaining game that are worth emphasising: incomplete information and renegotiations. First, as is commonly assumed in the literature on vertical contracting but not explicitly depicted in Figure 1, our bargaining game is one of incomplete information. ${ }^{14}$ In particular, agents do not know the prices and quantities agreed upon in earlier negotiations that they did not participate in. These cannot be observed ex post; eliminating the ability to agree to contracts contingent upon the particular pricing outcomes of other negotiations. Thus, a negotiating pair can

${ }^{12}$ As is well-known, holding the outcomes of other negotiations as fixed, as the probability of a breakdown becomes arbitrarily small, a pair bargaining in this fashion will agree on the Nash bargaining solution.

${ }^{13}$ As will be demonstrated below, as in SZ, the original order does not matter for surplus generated or

${ }_{14}$ See, for example, HT, O'Brien and Schaffer (1992), McAfee and Schwartz (1994), Rey and Tirole
(19) 
engage in secret discounting that enhances the future competitive position of a downstream firm at the expense of their rivals. Negotiating pairs anticipate such effects impacting on their own equilibrium agreements.

Given this, agents will form beliefs about the outcomes of negotiations they do not participate in. To refine the set of possible equilibrium outcomes, we adopt the commonly used assumption that agents hold passive beliefs regarding the prices agreed upon in earlier negotiations. ${ }^{15}$ Under passive beliefs, an agent's beliefs about the outcomes of other negotiations are not revised by an unexpected price offer.

Second, in our bargaining game, once negotiations have commenced, a supply agreement will only take place in equilibrium if the joint payoff to the upstream and downstream pair exceeds what each might receive if an agreement never takes place. ${ }^{16}$ This is reasonable as a non-agreement is possible and the parties should, given the lumpsum transfer, be able to jointly earn more from agreement than not.

But what payoffs will the parties expect to receive if an agreement never takes place? Answering this requires specifying what occurs in remaining negotiations and to past agreements in the event of such a breakdown. First, given that the breakdown is permanent, it is reasonable to assume that such events are common knowledge. Second, it is plausible that, in reality, remaining supply agreements might be impacted upon by such an event. For example, if a downstream firm were supplied by both upstream firms, but the relationship with one broke down irrevocably, the remaining upstream firm might eventually be able to negotiate a more favourable agreement. We take this into account by assuming that in the event of a breakdown all other supply agreements can be renegotiated.

This has two interpretations, both of which turn out to have equivalent

\footnotetext{
(1997) and Segal (1999).

${ }^{15}$ The assumption of passive beliefs arises naturally when supply negotiations occur simultaneously or downstream firms are not able to observe the precise sequence of negotiations (Hart and Tirole, 1990). While, for notational convenience, we assume here that agents know the negotiation order, our model and environment could easily accommodate a situation where this was unverifiable. See McAfee and Schwartz (1994) and Rey and Tirole (2003) for detailed discussions. SZ also implicitly assume passive beliefs when analysing the equilibrium outcome of their extensive form game. Recently, Segal and Whinston (2003) and Rey and Verge (2002) have constructed models relaxing the passive beliefs assumption to largely confirm the robustness of results in the vertically contracting literature.

${ }^{16}$ This assumption is an axiom in the bilateral oligopoly bargaining model of Inderst and Wey (2003). Here it is an outcome of the particular extensive form bargaining game that fixes the probability that an
} 
implications. First, the renegotiation option may arise because, say, an upstream firm can hold up its downstream customer by refusing to honor the past agreement. If that agreement is too costly to enforce, it will be renegotiated. Operationally, this amounts to an assumption that parties cannot jointly commit to refrain from renegotiations following a breakdown in supply relationships by others. ${ }^{17}$ This lack of commitment is a common assumption in the literature on incomplete contracts and the property rights theory of the firm. ${ }^{18}$ It is generally applied in environments in which price contracts are renegotiated more frequently than the market or ownership structure changes. ${ }^{19}$

Second, parties may negotiate contracts that take into account contingencies relating to the breakdown of other supply agreements. Such contracts will specify price and quantity terms if no breakdown were to occur elsewhere but also how those terms would be adjusted if supply relationships involving other pairs were to dissolve. ${ }^{20}$ Below we demonstrate that there is an equilibrium where the contingent supply terms are the same as the terms that would be renegotiation-proof in the event that contingent contracts were not binding. However, as in the contingent contract case of Inderst and Wey (2003), and in contrast to equilibria arising in the incomplete contracts case, this equilibrium may not be unique. For this reason, the specific extensive form game reflects the first interpretation but we will demonstrate equivalence to the latter interpretation for key results below.

Because agreements are renegotiated following breakdowns or are made

exogenous breakdown may occur during bilateral negotiations.

${ }^{17}$ Alternatively, parties could write contingent contracts with terms contingent upon the structure of supply agreements elsewhere but those contingent contracts must be renegotiation proof.

${ }_{8}^{8}$ This literature begins formally with the work of Grossman and Hart (1986) but has its antecedents in Klein, Crawford and Alchian (1978) and Williamson $(1975,1985)$. Hart $(1995)$ reviews the theory of contractual incompleteness based on the costliness of writing contracts to deal with every contingency. Hart and Moore (1988) and Dewatripont (1989) develop the theory with regard to an inability to commit not to renegotiate contract terms ex post.

${ }^{19}$ There are two reasons why an assumption of contractual incompleteness is reasonable in the context of this paper on vertical integration. First, the basic idea is that it is costly to write contracts contingent on small probability events. As will be demonstrated below, we treat a permanent breakdown in negotiations as an extremely unlikely event (with an infinitesimally small probability); falling into the class of contingencies that would not be contractible. Second, our model of vertical integration is based on the property rights framework that presumes that all negotiations take place after asset ownership is determined; that is, supply negotiations cannot be made contingent upon ownership structures. In this respect, by preventing contracts being contingent upon the structure of supply agreements we are treating changes in these in a symmetric manner to changes in ownership.

${ }^{20}$ Again, such contingent contracts an axiom in the bargaining model of Inderst and Wey (2003). 
contingent upon them, subgame perfection implies that all players take disagreement payoffs as given in their current negotiations. ${ }^{21}$ The irrevocability of breakdowns means that following this, the game will never return to the current "node of the game," the set of negotiations currently underway. Therefore, agents cannot credibly choose a postbreakdown strategy that will improve their payoff in the current negotiations. Instead, after a breakdown they will follow the strategy that maximizes payoffs in postbreakdown negotiations.

The combination of our assumptions regarding passive beliefs and what happens following breakdowns elsewhere serves to simplify the multi-person bargaining game dramatically. In particular, we can analyze each bilateral negotiation, taking the outcomes of other negotiations as given. This allows us to derive explicit closed form solutions in an otherwise general economic environment (in terms of demand and production technologies); making the analysis of bilateral oligopoly quite tractable. In addition, as will be demonstrated below, our solution concept replicates cooperative bargaining concepts (such as the Shapley value and its extensions by Myerson) only in certain circumstances. In particular, when there are competitive externalities, our solution is novel in that it does not arise in cooperative game theory. ${ }^{22}$

\section{$3 \quad$ Bargaining and Integration with No Externalities}

We begin by assuming, in this section, that there are no competitive externalities downstream. ${ }^{23}$ That is, for each $D_{i}, \pi_{i}\left(q_{i A}, q_{i B}, q_{-i A}, q_{-i B}\right)=\pi_{i}\left(q_{i A}, q_{i B}, 0,0\right) \equiv \pi_{i}\left(q_{i A}, q_{i B}\right)$ for all $\left(q_{-i A}, q_{-i B}\right)$. This may arise if downstream firms sell distinct products using a similar set of inputs, sell products in different geographical markets, or sell highly differentiated

${ }^{21}$ Alternatively, the disagreement payoffs are governed by the contingencies negotiated by others are not observed across negotiating pairs. Hence, given passive beliefs, they cannot be impacted upon by the negotiating pair

${ }_{22}$ For example, Inderst and Wey (2003) use an axiomatic approach to analyze bilateral oligopoly. In so doing, they motivate use of the Shapley value. As their environment presumes that there are no competitive externalities, our bargaining game can be viewed as providing a non-cooperative foundation for their approach and a demonstration of how this would extend to an environment where downstream firms compete with one another.

${ }^{23}$ To clarify, there are still externalities between negotiations in that an agreement by one pair impacts upon upstream costs faced in another. However, we demonstrate that such externalities are internalized. 
products. ${ }^{24}$ As will be demonstrated, this case allows us to isolate the impact of vertical integration on each agent's bargaining position - holding efficiency considerations fixed.

\section{$3.1 \quad$ Non-Integration}

To build intuition, we first examine the case of non-integration when there is upstream competition. Under non-integration, all four assets are separately owned by their respective managers, who can potentially negotiate with any vertically related manager. As we will see, this is not the case under integration.

Given the assumption of passive beliefs we can solve for the equilibrium payoffs of each agent. Moreover, we can demonstrate that the outcome is efficient in that industry profits are maximized.

Proposition 1. In any perfect Bayesian equilibrium with passive beliefs, $\left(q_{1 A}, q_{2 A}, q_{1 B}, q_{2 B}\right)$ are such that $\pi_{1}\left(q_{1 A}, q_{1 B}\right)+\pi_{2}\left(q_{2 A}, q_{2 B}\right)-c_{A}\left(q_{1 A}, q_{2 A}\right)-c_{B}\left(q_{1 B}, q_{2 B}\right)$ is maximized. Each agent receives their payoff as given in Table 1.

The proof is in the appendix. Notice that this result is independent of the precise ordering of pairs in sequential negotiations. ${ }^{25}$

The intuition for efficiency is subtle, given the interactions between the negotiations of each pair of agents. As depicted in Figure 2(a), under non-integration, there are potentially four pairs of negotiations. Each negotiation involves Nash bargaining where the pair chooses their respective supply quantity to maximize their bilateral payoff. For example, $U_{A}$ and $D_{1}$ would choose $q_{1 A}$ to maximize:

$$
\pi_{1}\left(q_{1 A}, q_{1 B}\right)-\tilde{p}_{1 B}-\Phi_{1 A}+\tilde{p}_{2 A}-c_{A}\left(q_{1 A}, q_{2 A}\right)-\Phi_{A 1}
$$

while $\tilde{p}_{1 A}$ would satisfy:

\footnotetext{
${ }^{24}$ This case has been a common focus of the literature on strategic vertical integration (Bolton and Whinston, 1993), the role of exclusive contracts (see, for example, Segal and Whinston, 2000) as well as competition in buyer-seller networks (Jackson and Wolinsky, 1996; Kranton and Minehart, 2001). In work contemporary with the present paper, Inderst and Wey (2003) and Bjornerstedt and Stennek (2001) also provide an analysis of the no competitive externalities case under conditions of bilateral oligopoly.

${ }^{25}$ We could weaken that passive beliefs requirement and consider 'wary beliefs.' In this case, parties anticipate that later negotiation behavior will be adjusted according to deviations in earlier negotiations. (see McAfee and Schwartz, 1994). This weaker assumption, however, does not result in the same outcome as passive beliefs when there are competitive externalities. In that case, the order of negotiations does matter, complicating considerably the notational complexity of the paper but without any change in the qualitative results regarding vertical integration.
} 


$$
\pi_{1}\left(q_{1 A}, q_{1 B}\right)-\tilde{p}_{1 A}-\tilde{p}_{1 B}-\Phi_{1 A}=\tilde{p}_{1 A}+\tilde{p}_{2 A}-c_{A}\left(q_{1 A}, q_{2 A}\right)-\Phi_{A 1}
$$

where $\Phi_{i j}$ and $\Phi_{j i}$ represent the payoffs $D_{i}$ and $U_{j}$ expect to receive in the renegotiation subgame triggered by a breakdown in their negotiations; by subgame perfection, these are taken as given. The remaining pricing terms either form the subject of a previous agreement earlier in the bargaining sequence (in which case their terms are given by the assumption of passive beliefs) or anticipate the negotiations of pairs further in the sequence. In that case, we can demonstrate that when anticipated outcomes are substituted into (1), the only term involving $q_{1 A}$, taking into account the envelope theorem, is a linear function of $\pi_{1}\left(q_{1 A}, q_{1 B}\right)-c_{A}\left(q_{1 A}, q_{2 A}\right)$. Thus, $q_{1 A}$ is always chosen to maximize industry profits.

In terms of distribution, the equilibrium payoffs in Table 1 are obtained by resolving the equivalent of (2) for all pairs and all subgames. They represent the Shapley values of each respective agent given the allocation of assets among them. ${ }^{26}$ While other analyses of bilateral oligopoly have derived Shapley value outcomes using axiomatic bargaining treatments ours is based on an explicit extensive form. In addition, while we demonstrated in the Appendix that the equilibrium is equivalent to situations (such as assumed by Inderst and Wey (2003)) that require supply agreements to specify pricing arrangements that would arise for every industry configuration, this interpretation is not unique. The same outcome arises when we do not allow negotiating agents to commit to supply arrangements contingent on exit or the severing of any supply relationship in the industry.

What is most significant about this distribution is its coalitional form; where each agent's payoff depends on industry profits generated under various alternative supply configurations. Thus, if, say, there is a breakdown between $U_{A}$ and $D_{l}$, bargaining proceeds between the remaining pairs on the basis that no supply can occur between them. Interestingly, as was noted by Jackson and Wolinsky (1996) for the cooperative game context, only certain types of supply configurations actually enter into the resulting

${ }^{26}$ This mirrors the finding of SZ. A similar type of result drives Stole and Zwiebel (1998). Their paper considers the impact of a horizontal merger amongst non-competing firms on intra-firm bargaining with
workers. A horizontal merger means that workers connected previously to one another through two firms, will be connected directly to the merged entity. Stole and Zwiebel (1998) show that this may improve or harm their bargaining position depending upon the nature of cost savings from the merger. 
payoff. Specifically, supply configurations where one supply relationship has been severed but otherwise all firms remain connected (in a graph-theoretic sense) do not appear in payoffs; those terms are relevant in bargaining off the equilibrium path but cancel out in the equilibrium payoffs because of the game's recursive structure. This simplifies the form of the payoffs and eliminates the need to make strong assumptions about outcomes where links between groups of agents are only partially severed.

\subsection{Vertical Integration}

Vertical integration involves a change in asset ownership between an upstream and a downstream manager. We will focus here on vertical integration between $U_{A}$ and $D_{1}$. This may involve forward integration (FI) whereby $U_{A}$ acquires $D_{1}$ 's assets or backward integration (BI) where $U_{A}$ 's assets are acquired by $D_{1} \cdot{ }^{27}$ In each case, as in the property rights literature, the acquirer becomes the residual claimant to the earnings of an asset and has residual control rights as to what it is used for (Grossman and Hart, 1986; Hart and Moore, 1990). However, each manager continues to be essential for the productive use of the asset.

To illustrate what changes in ownership mean in the present context, suppose $U_{A}$ integrates forward by purchasing $D_{1}$ 's assets. The manager of the acquired $D_{1}$ receives a transfer payment, $\tilde{t}_{1 A}$, while the profits from its asset, $\pi_{1}\left(q_{1 A}, q_{1 B}\right)-\tilde{t}_{1 A}-\tilde{p}_{1 B}$, accrue to the new owner, $U_{A}{ }^{28}$ Importantly, as depicted in Figure 2(b), $U_{A}$ rather than $D_{1}$ negotiates a supply agreement with $U_{B}$ for the supply of inputs to $D_{1}$. This is because the residual control rights of the downstream asset have been transferred to $U_{A}$. Thus, in the event of a breakdown in negotiations between $U_{A}$ and the manager of $D_{1}$, no supply will occur between $U_{B}$ and $D_{1}$.

${ }^{27}$ There is a third option characterized by some form of joint ownership. As there is an issue with regard to how that form of ownership might operate in this setting (see Bolton and Whinston, 1993), we do not consider it here.

${ }^{28}$ Note that while it may be possible to offer the manager of the acquired asset a share of the profits of the merged entity, as long as this is less than 50 percent, it is reasonable to suppose that the new owner has residual control rights. This means that agent can exclude the old owner and anyone else from access to the residual control rights. This means that agent can exclude the old owner and anyone else from access to the asset. Thus, the old owner's compensation will be up for negotiation. As shown clearly by Aghion and Hence, we adopt the convention here of modeling a transfer of ownership as a complete transfer of equity to the new owner. 
What this means is that a breakdown between $U_{A}$ and the manager of $D_{1}$ has a deeper impact upon $U_{B}$ and $D_{2}$. While, under non-integration, such a breakdown would still mean that $D_{1}$ could continue to receive inputs from $U_{B}$, under FI, this would no longer occur. In this case, $U_{B}$ would be left with $D_{2}$ as its sole source of demand. FI thus eliminates the possibility of $U_{B}$ being the only supplier of $D_{1}$, thereby weakening its bargaining power. For the same reason, FI improves the bargaining position of $D_{2}$ as it increases the chances it will not have to compete with $D_{1}$ for $U_{B}$ 's input.

In this environment, it can be demonstrated - along the same lines as in the proof of Proposition 1 - that integration (BI or FI) will only affect the distribution of surplus between agents and not the overall surplus generated. As in non-integration, this occurs because, under passive beliefs, each negotiating pair chooses its respective quantity in a way that does not impact on the pricing and quantity terms of other negotiations. Thus, the supply quantities chosen continue to maximize industry profits.

The payoffs contained in Table 1 show how distribution changes following integration. The critical feature to note about the effect of integration is that it rules out the participation of an asset's manager in a coalition that does not include the owner. When $U_{A}$ owns $D_{1}$ (that is, forward integration FI), the payoff $\Pi\left(\overline{D_{1} D_{2} U_{B}}\right)$ becomes $\Pi\left(\overline{D_{2} U_{B}}\right)$, and the payoff $\Pi\left(\overline{D_{1} U_{B}}\right)$ becomes 0 . When $D_{1}$ owns $U_{A}$ (that is, backward integration BI), the payoff $\Pi\left(\overline{D_{2} U_{A} U_{B}}\right)$ becomes $\Pi\left(\overline{D_{2} U_{B}}\right)$, and the payoff $\Pi\left(\overline{D_{2} U_{A}}\right)$ becomes 0 . In each case, integration diminishes the bargaining position of one or both of the non-integrated firms and, as is depicted in the last two rows of Table 1, this raises $U_{A}$ and $D_{1}$ 's joint payoff from integration over non-integration by $\frac{1}{6}\left(\Pi\left(\overline{D_{1} D_{2} U_{B}}\right)-\Pi\left(\overline{D_{2} U_{B}}\right)\right)$ for FI and $\frac{1}{6}\left(\Pi\left(\overline{D_{2} U_{A} U_{B}}\right)-\Pi\left(\overline{D_{2} U_{B}}\right)\right)$ for BI.

Comparing these two changes in payoffs, notice that FI will be chosen over BI if and only if $\Pi\left(\overline{D_{1} D_{2} U_{B}}\right)>\Pi\left(\overline{D_{2} U_{A} U_{B}}\right)$. That is, FI is favoured as an instrument for improving joint bargaining power precisely when upstream firms are closer substitutes than downstream firms. ${ }^{29}$ In other words, the acquiring firm comes from the more

${ }^{29}$ Upstream firms are closer substitutes when $\Pi\left(\overline{D_{2} U_{A} U_{B}}\right) \rightarrow \Pi\left(\overline{D_{2} U_{j}}\right)$ while downstream firms are closer substitutes (in the eyes of upstream firms) when $\Pi\left(\overline{D_{1} D_{2} U_{A}}\right) \rightarrow \Pi\left(\overline{D_{i} U_{A}}\right)$. This does not, however, mean 
competitive vertical segment. This is because integration eliminates an option for the acquirer's competitor, an option that is valuable precisely because firms in the other vertical segment are not close substitutes from their perspective (and therefore that segment is less competitive). For example, forward integration means that $U_{B}$ loses an option to supply both downstream firms and this loss is costly when supplying both is relatively valuable. Consequently, the non-integrating firm that suffers the greatest harm from integration is the firm that is in the same segment as the acquiring firm (i.e., $D_{2}$ under $\mathrm{BI}$ and $U_{B}$ under $\mathrm{FI}$ ).

Importantly, our results here generalize HT's 'scarce needs' and 'scarce supplies' motives for vertical integration, by allowing for upstream costs to lie between the extremes of constant and backwards L-shaped marginal costs. To see this, observe that when upstream marginal costs are constant and symmetric (that is, there are 'scarce needs' as industry supply is perfectly elastic), $D_{1}$ and $U_{A}$ have no incentive for BI but a positive incentive for FI. In this case, $D_{2}$ 's payoff is unchanged and rents shift entirely from $U_{B}$. In contrast, when upstream firms are capacity constrained and downstream firms are perfectly substitutable ${ }^{30}$ (that is, there are 'scarce supplies'), there is no incentive for FI but a positive incentive for BI. In that case, it is $U_{B}$ 's payoff that is unchanged by integration with the impact being borne entirely by $D_{2}$. This accords with the general findings of HT. ${ }^{31}$ However, we have derived these motives for vertical integration in a model where bargaining position is determined by the characteristics of possible breakdown market structures rather than an exogenous parameter. We demonstrate below that these motives are preserved when competitive externalities are considered.

that downstream firms sell products that are close substitutes from a consumer perspective. It might simply mean that upstream firms have convex cost technologies.

${ }^{30}$ In the no externalities case, this would arise if $\partial \pi_{i} / \partial q_{i j}$ were constant for any quantity smaller than total upstream capacity. In HT, they assume that downstream outputs are perfect substitutes that also make those firms perfect substitutes. We consider this case in Section 4 below.

${ }^{31}$ Strictly speaking, while HT find that only $D_{2}$ is harmed under 'scarce supplies,' in their 'scarce needs' model both non-integrated firms were harmed by integration. In our model, when upstream costs lie between these two extremes, we also find the both $D_{2}$ and $U_{B}$ are harmed by integration. 
As the focus of this paper is the change in the effect of vertical integration as upstream competition is introduced, we need to take care in specifying the upstream monopoly case. ${ }^{32}$ In particular, we require the set of productive assets in the industry to be the same between the two cases as well as the characteristics of any human capital. This means that we cannot simply take the two upstream assets and combine them under a single owner, as one of the assets will be managed by an individual with important human capital. As with vertical integration, that agent cannot be replaced and so will have some bargaining power in negotiations with the owner of upstream assets.

The only difference between the outcomes under upstream monopoly as compared with upstream competition is in the distribution of the surplus between agents. Industry profits are maximized under the same logic as Proposition 1 and these profits are the same as under upstream competition, as the characteristics of resources in the industry are unchanged. In contrast, the payoffs of individual agents - listed in Table 1 - are different under upstream monopoly.

The negotiating relationships for upstream monopoly are depicted in Figure 3(a). In comparison with the upstream competition case, there are only three relevant negotiations as there is only a single firm negotiating the supply of inputs to downstream firms. What this means is that if negotiations between the upstream monopolist (chosen to be $U_{A}$ ) and a downstream firm break down, the downstream firm exits the industry.

As before, we consider vertical integration between $U_{A}$ and $D_{1}$. The changed bargaining relationships are depicted in Figures 3(b) and 3(c) for the cases of forward and backwards integration, respectively. Notice that, under forward integration, the change in residual control rights implies no change in the bargaining relationships. This means that forward integration will yield exactly the same payoffs as non-integration.

In contrast, the changes in bargaining relationships under backwards integration are quite extensive (see Figure $3(\mathrm{c})$ ). In this situation, $D_{1}$ purchases $U_{A}$ 's assets. This makes $D_{1}$ the owner of its assets and those of $U_{A}$ and $U_{B}$. It will negotiate with both of

${ }^{32}$ All of the results regarding vertical integration in this sub-section would similarly hold if we had a downstream monopsony rather than upstream monopoly. There would, however, be a difference in results when we include competitive externalities downstream. 
those managers. Hence, backwards integration allows some market structures to be possible relative to the non-integration case. In particular, it is now possible for $D_{1}$ to rely solely on supply from $U_{B}$, because $U_{B}$ 's manager can still supply $D_{1}$ if negotiations break down between $D_{1}$ and $U_{A}$ 's manager. The implication is that BI may improve $U_{B}$ 's bargaining position. ${ }^{33}$

Backward integration is preferred to the status quo - or, equivalently, FI - if $\Pi\left(\overline{D_{2} U_{A} U_{B}}\right)>\Pi\left(\overline{D_{1} D_{2} U_{B}}\right)$; this is the same condition as under upstream competition. In other words, $\mathrm{BI}$ is preferable if upstream assets are relatively less substitutable than downstream assets. Otherwise, BI may not be privately desirable as it improves the bargaining power of $U_{B}$ whose productive role is otherwise similar to $U_{A}$. Thus, as in the upstream competition case, the acquiring firm comes from the segment that is relatively competitive and not from the monopoly segment as is the presumption of conventional wisdom.

\subsection{Comparison of Upstream Competition and Upstream Monopoly}

We are now in a position to compare the incentives for vertical integration in upstream monopoly with those for upstream competition, based on pure bargaining effects. Recall that the payoff to FI relative to BI is determined by the same condition in upstream monopoly and upstream competition; so we can look at FI and BI in turn, using the results in Table 1.

For FI, the comparison is clear: there is no incentive for FI under upstream monopoly, but a positive incentive for FI under upstream competition. FI confers additional market power on the upstream firm, by ruling out options for the other upstream firm; but under upstream monopoly, this has already been achieved.

For BI, it is easy to see that it too will improve the joint payoff to $U_{A}$ and $D_{1}$ by more under upstream competition than under upstream monopoly, as $\Pi\left(\overline{D_{1} D_{2} U_{B}}\right) \geq \Pi\left(\overline{D_{2} U_{B}}\right)$. BI eliminates the possibility of a $D_{2}$ monopsony facing the upstream firms. Under upstream competition, BI also increases the chance of a bilateral

${ }^{33}$ For example, when $U_{A}$ and $U_{B}$ are perfect substitutes (i.e., symmetric with linear costs), $U_{B}$ obtains no rents under non-integration and positive rents under backwards integration. 
monopoly between $U_{B}$ and $D_{2}$, whereas under upstream monopoly the possibility of a $U_{B}$ monopoly is reintroduced.

Thus, from a pure bargaining perspective, integration has a higher private return under upstream competition than under upstream monopoly. The reason for this is that the benefits of integration flow from harming agents outside of the proposed merger thereby redistributing rents in favour of the insiders. Under upstream monopoly, outsiders either do not have their bargaining position change, or in some cases can potentially improve their negotiating relationships with insiders. For upstream competition, integration always removes possible market structures that may have been of benefit to outsiders. Hence, the incentive for integration is stronger under upstream competition. If vertical integration involved a fixed cost (in terms of foregone investment, or transactions costs), integration would be more likely under upstream competition. ${ }^{34}$

\section{Competitive Externalities}

The previous section demonstrates that incentives for strategic vertical integration can be higher under upstream competition than upstream monopoly. In the no externalities case, however, production is unchanged following integration so industry profits are always maximized. Integration served only to change distribution in ways that were different, depending upon the degree of upstream competition. When there are competitive externalities, the distributional (or bargaining) consequences of vertical integration are largely identical. What differs is the level of production, meaning that integration has welfare consequences. As we demonstrate in this section, vertical integration can lead to higher downstream prices and increased deadweight losses, as in the contracting externalities literature. Critically, however, the industry profits generated by vertical integration differ between upstream competition and monopoly. In this section, we explore how upstream competition affects the welfare losses from integration.

${ }^{34}$ Given that the payoffs of at least one non-integrated firm is reduced by integration, there is also a possibility that integration may induce exit. In this case, there will be a direct welfare consequence of integration. In HT exit is the only way in which distribution effects can occur. Instead, here we have provided a context in which exit does not occur (i.e., there are no fixed costs) but distributional effects from vertical integration still arise 
The contracting externalities literature typically considers a monopolist selling to downstream firms producing identical goods. ${ }^{35}$ The monopolist makes take-it-or-leave-it offers to each firm in turn. If it were to sell the profit-maximizing quantity to the first, it would have an incentive to "secretly discount" (i.e., sell more than the profit-maximizing quantity, at a discount) to other downstream firms, as those later offers would not internalize any externality imposed on contracts already signed. For this reason, firms will not accept a contract consistent with industry profit maximization. If prices and quantities are unobservable, and if agents hold passive beliefs, implying that they do not revise their beliefs about prices and quantities in other contracts when they observe behavior that is off the equilibrium path, the only perfect Bayesian equilibrium is for the monopolist to offer Cournot quantities to all firms. In other words, each negotiating pair maximizes their profits, taking the negotiated quantity in the other agreement as given. ${ }^{36}$

A similar set of outcomes arises in our bargaining environment. Bilateral Nash bargaining implies that each $q_{i j}$ is chosen to maximize $\pi_{i}()-.c_{j}($.$) , taking as given$ quantities chosen in other negotiations (by passive beliefs). When there were no competitive externalities, this choice did not impact upon the outcome of other negotiations; therefore each choice maximized industry profits. Similarly, in market structures where only one downstream firm is present, industry profits will still be maximized, as there are no competitive externalities. However, total industry profits will not be maximized overall when both downstream firms are present, as each negotiation imposes externalities on others. Let $\hat{\Pi}($.) represent equilibrium industry profits in that case. The following proposition summarises the equilibrium outcome:

Proposition 2. There exists a unique perfect Bayesian equilibrium with passive beliefs, under non-integration, regardless of whether there is upstream competition or monopoly, in which $\hat{\Pi}\left(\overline{D_{1} D_{2} U_{A} U_{B}}\right)$, $\hat{\Pi}\left(\overline{D_{1} D_{2} U_{A}}\right)$ and $\hat{\Pi}\left(\overline{D_{1} D_{2} U_{B}}\right)$ are at their Cournot duopoly levels when upstream inputs are supplied on the basis of industry upstream marginal cost. For all other industry configurations, $\hat{\Pi}()=.\Pi($.$) .$

${ }^{35}$ See HT, Rey and Tirole (1997), O'Brien and Shaffer (1992) and McAfee and Schwartz (1994).

${ }^{36}$ The role of passive beliefs here is to neutralize the effect of bargaining order on outcomes (McAfee and Schwartz, 1994). 
As we do not rule out quantity commitments, our model yields the same conclusion reached in the contracting externalities literature, that under passive beliefs, Cournot outcomes will result. What is interesting here is that this is achieved despite the fact that there are two independently owned upstream assets, yielding the same outcome as an upstream monopoly where both assets are commonly owned. ${ }^{37}$ Interestingly, this implies that an upstream (horizontal) merger does not change retail prices and welfare downstream in this setting. ${ }^{38}$ Because there is no negotiation involving residual claimants on the returns of both downstream assets, there is no negotiation in which the impact of a supply choice on both downstream firms is considered. Instead, in each negotiation the quantity supplied to one downstream firm is chosen holding supply to the other constant - yielding a Cournot equilibrium.

EXAMPLE: Suppose that both downstream firms face linear demand, $p_{i}=1-\left(q_{i A}+q_{i B}\right)-\gamma\left(q_{-i A}+q_{-i B}\right) \quad(1 \geq \gamma) \quad$ and have cost functions $c_{i}\left(q_{i A}, q_{i B}\right)=\theta\left(q_{i A}-q_{i B}\right)^{2} \quad$ (with $\left.1 \geq \theta \geq 0\right)$ while upstream firms $(j=A$ or $B$ ) have symmetric cost functions, $c_{j}\left(q_{1 j}+q_{2 j}\right)=\left(q_{1 j}+q_{2 j}\right)^{2}$. The unique equilibrium under both upstream competition and monopoly involves both downstream firms being supplied $\hat{q}_{i j}=\frac{1}{8+2 \gamma}$ by both upstream firms, generating profits of $\Pi\left(D_{1} D_{2} U_{A} U_{B}\right)=\frac{4}{(4+\gamma)^{2}}$; greater than the fully integrated monopoly outcome of $q_{i j}^{*}=\frac{1}{8+4 \gamma}$ with $\Pi\left(\overline{D_{1} D_{2} U_{A} U_{B}}\right)=\frac{1}{4+2 \gamma}$. Similarly, in this situation, $\hat{\Pi}\left(\overline{D_{1} D_{2} U_{j}}\right)=\frac{2(3+\theta)}{(6+2 \theta+\gamma)^{2}}$ with $\hat{q}_{i j}=\frac{1}{6+2 \theta+\gamma}$.

What happens when $D_{1}$ and $U_{A}$ integrate? First, as in the no externality case, this eliminates certain market structures depending upon whether there has been FI or BI. Second, for those market structures that remain possible, equilibrium industry profits are unchanged for all market structures where one or more of $D_{1}, D_{2}$ and $U_{A}$ are not present. That is, a change in equilibrium profits following integration requires the presence of both $D_{1}$ and $U_{A}$, and it is only where $D_{2}$ is also present that industry profits are not necessarily maximized under non-integration and integration alike.

Third, the impact on equilibrium outcomes from integration is the same under

${ }^{37}$ That is, suppose, as do HT, that both upstream firms have constant marginal costs but that $U_{B}$ 's is higher than $U_{A}$ 's. In this case, under upstream monopoly, $U_{A}$ would never choose to supply using the other upstream asset. And under upstream competition, the Cournot equilibrium would involve supply purely may still be paid in equilibrium. 
both BI and FI. In each case, integration implies that the residual claimant on the profits of $D_{1}$ is the one negotiating the supply from $U_{A}$ to $D_{2}$. Under both FI and BI, in negotiations over $q_{2 A}$, the negotiated supply quantity maximizes $\pi_{1}()+.\pi_{2}()-.c_{A}($.$) . This$ is because the residual claimant on $D_{1}$ 's profits negotiates with $D_{2}$ over the supply from $U_{A}$ to $D_{2}$. Nonetheless, negotiations that are internal to the integrated firm will still involve supply quantities chosen to maximize $\pi_{i}()-.c_{j}(.) . D_{2}$ does not participate in those negotiations and hence, the impact on its profits is not considered.

Fourth, there is a difference between the impact of integration in the upstream competition and monopoly cases. Under upstream competition, negotiations over $q_{2 B}$ will still maximize $\pi_{2}()-.c_{B}($.$) whereas, under upstream monopoly,$ $\hat{q}_{2 B} \in \arg \max _{q_{2 B}} \pi_{1}()+.\pi_{2}()-.c_{B}($.$) . The fact that competitive externalities are$ internalized in two negotiations rather than one suggests that integration will allow an upstream monopolist to more easily restrict output and raise prices downstream. Given the general nature of profit and cost functions (and potential asymmetries between firms) assumed thus far, it is not possible to provide a simple proof of this.

Nonetheless, by imposing further restrictions, we can characterise the effects of integration on industry profits explicitly.

Proposition 3. Let $\hat{\Pi}_{U C}($.$) and \hat{\Pi}_{U M}($.$) denote industry profits in any perfect Bayesian$ equilibrium with passive beliefs under integration by $D_{1}$ and $U_{A}$, for upstream competition and monopoly respectively. Assume that (1) $D_{1}$ and $D_{2}$ are symmetric and are indifferent to the source of supply; (2) each $c_{j}$ has symmetric and weakly concave isoquants (for given total cost) in $\left(q_{1 j}, q_{2 j}\right)$. Then,

(i) Industry profits are unchanged following integration under upstream competition; i.e., $\hat{\hat{\Pi}}_{U C}\left(\overline{D_{1} D_{2} U_{A} U_{B}}\right)=\hat{\Pi}\left(\overline{D_{1} D_{2} U_{A} U_{B}}\right)$;

(ii) If $D_{1}$ and $D_{2}$ sell products that are perfect substitutes, then all externalities are internalized under market structures with an upstream monopoly; i.e.

$$
\hat{\hat{\Pi}}_{U M}\left(\overline{D_{1} D_{2} U_{A} U_{B}}\right)=\Pi\left(\overline{D_{1} D_{2} U_{A} U_{B}}\right) \text { and } \hat{\hat{\Pi}}_{U M}\left(\overline{D_{1} D_{2} U_{A}}\right)=\hat{\hat{\Pi}}_{U C}\left(\overline{D_{1} D_{2} U_{A}}\right)=\Pi\left(\overline{D_{1} D_{2} U_{A}}\right) \text {. }
$$

Proposition 3 provides a sharp characterization of the outcomes in an environment where upstream competition is very strong (as upstream inputs are perfectly substitutable from the point of view of downstream firms). The concavity of upstream cost isoquants means

${ }^{38}$ If there were externalities amongst upstream firms, this would no longer be the case. 
that it is (weakly) cost minimizing for each upstream firm to supply a single downstream firm, under non-integration as well as integration. ${ }^{39}$ In the presence of upstream competition, therefore, all integration does is change supply relations, without changing the actual production or surplus generated. ${ }^{40}$ This leads to the interesting result that if a dedicated supply flow is optimal, there is no change in industry profits following integration (Result (i)). ${ }^{41}$

When downstream firms are perfect substitutes, we can further characterize the results. When there is an upstream monopolist- either $U_{A}$ and $U_{B}$ are owned by the same manager, or $U_{B}$ has exited the market following breakdowns in negotiation, leaving $U_{A}$ alone in the market -integration leads to foreclosure of the non-integrated firm, $D_{2}$. The monopoly quantity is supplied to $D_{1}$, and profits are thereby maximized. ${ }^{42}$ It is important to note, however, that while it appears that foreclosure occurs here - as the independent downstream firm receives no inputs from its integrated rival - this does not necessarily mean that it receives no payment integrated firm; a point we discuss in more detail when we consider the distribution of surplus below. ${ }^{43}$

When $D_{1}$ and $D_{2}$ are not perfect substitutes, upstream firms reduce their supply to $D_{2}$ but do not necessarily foreclose. This is precisely the monopolization effect from integration first identified by HT that arises because the integrated firm internalizes its own competitive externality when negotiating with outside parties. Industry profits are not perfectly maximized, in general, because the integrated firm does not take into account the externality it imposes on $D_{2}$; something borne out in our running example.

When it comes to integration under upstream competition, however, the impact of integration on overall profits is, in general, ambiguous. The main reason for this is that, while an upstream monopolist will necessarily take actions that realize productive

${ }_{40}^{39}$ Proposition 2 demonstrates that cost minimization is achieved in equilibrium.

${ }^{40} \mathrm{HT}$ 's ex post monopolization case similarly found that there was no increase in industry profits following integration. This was because their case is a special case of Proposition 3 with linear isoquants.

${ }^{41}$ This also assists in comparing our results to those of other models in the literature. For instance, Chen (2001) assumes that there are switching costs associated with changing from dedicated suppliers. This means that inputs are not perfect substitutes for downstream firms; hence, integration has an impact on industry profits in Chen's model.

${ }_{42}$ Proposition 3 is stated more strongly than necessary, on this point: no assumptions on upstream firms are necessary. It is only necessary for downstream firms to be perfect substitutes.

${ }^{43} \mathrm{HT}$ also find similar supply flows and industry profit outcomes as in Proposition 3 . However, as will be discussed below, we do not find that this means that $D_{2}$ is foreclosed in the traditional sense and forced to 
efficiency for upstream supply, there is no similar control in upstream competition. While this did not matter under non-integration, by creating incentives for $U_{A}$ to reduce its supply to $D_{2}$, integration creates the opposite incentives for $U_{B}$, who wants to expand supply to $D_{2}$. If downstream firms care about the source of input supply (i.e., do not view outputs from $U_{A}$ and $U_{B}$ as perfect substitutes), then these changes can increase industry costs and lead to a reduction in profits; a possibility we demonstrate in our running example below.

EXAMPLE (Continued): When $\theta=0$ (downstream firms are indifferent as to the source of input supply), vertical integration does not change the equilibrium outcome under upstream competition (as this involved each upstream firm supplying a single downstream firm); although $U_{A}$ will be the sole supplier of $D_{I}$ (thus,

$\hat{\hat{\Pi}}_{U C}\left(\overline{D_{1} D_{2} U_{A} U_{B}}\right)=\hat{\Pi}\left(\overline{D_{1} D_{2} U_{A} U_{B}}\right)$ ). For upstream monopoly, as all supply is controlled by the owner of $D_{l}$, the impact of any supply to $D_{2}$ on $D_{l}$ 's profits will be internalised for that decision. In addition, it is easy to confirm that both downstream firms will continue to be supplied (each from one downstream asset); although there will be a contraction of supply to $D_{2}$ relative to the non-integrated case (thus, $\left.\hat{\Pi}_{U M}\left(\overline{D_{1} D_{2} U_{A} U_{B}}\right)=\frac{32-40 \gamma+7 \gamma^{2}+4 \gamma^{3}}{2\left(2 \gamma^{2}+3 \gamma-8\right)^{2}}\right)$.

When $\theta>0$, integration changes industry profits under both upstream monopoly and upstream competition. In each case, there is an overall reduction in output with $D$ having a higher output than $D_{2}$. The following Figure 4(a) shows what happens to industry profits when $\theta=\frac{1}{2}$ and Figure 4(b) shows what happens to consumer surplus.

Note that $\hat{\hat{\Pi}}_{U M}\left(\overline{D_{1} D_{2} U_{A} U_{B}}\right)>\hat{\Pi}\left(\overline{D_{1} D_{2} U_{A} U_{B}}\right)>\hat{\hat{\Pi}}_{U C}\left(\overline{D_{1} D_{2} U_{A} U_{B}}\right)$ (for $\theta$ high enough) while for consumer surplus non-integration provides the best outcome and integration by an upstream monopolist is the worst. Overall total welfare follows the consumer surplus ranking. Nonetheless, consumers in $D_{I}$ 's $\left(D_{2}\right.$ 's) market are better (worse) off under integration with an upstream monopoly than the upstream competition case.

\section{(a) Industry Profits}

\section{Figure 4}

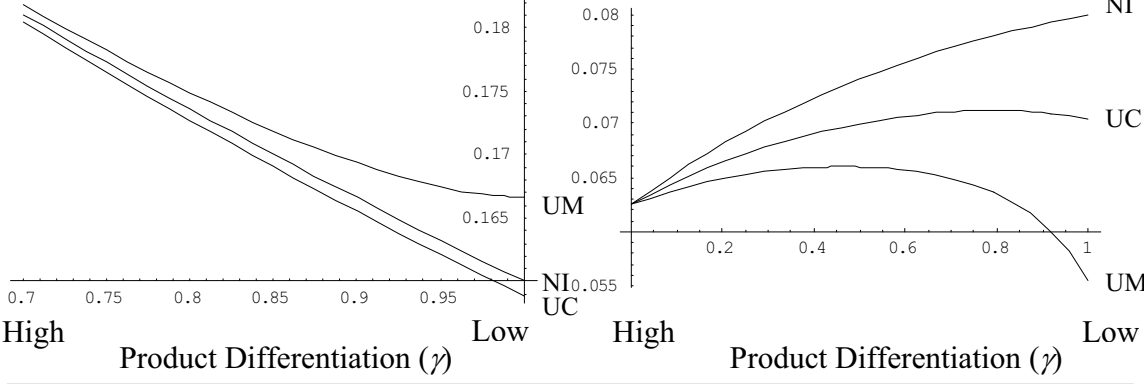

exit the industry.

${ }^{44}$ Defined as the unweighted sum of consumer surplus generated from both downstream products. 
In solving for the equilibrium payoffs under competitive externalities, there arises the important issue of what SZ term 'feasibility.' An equilibrium is feasible if there is no incentive for an individual party to precipitate a breakdown in any negotiating pair at any stage (i.e., in any market structure that might have emerged). Under competitive externalities feasibility cannot be guaranteed.

To see this, consider a situation where a single upstream firm, $U_{A}$, is negotiating with two non-integrated downstream firms. If both negotiating pairs agree then they divide up $\hat{\Pi}\left(D_{1} D_{2} U_{A}\right)$ with:

$$
\begin{aligned}
& v_{U_{A}}=\frac{1}{6}\left(2 \hat{\Pi}\left(D_{1} D_{2} U_{A}\right)+\Pi\left(D_{1} U_{A}\right)+\Pi\left(D_{2} U_{A}\right)\right), \\
& v_{D_{1}}=\frac{1}{6}\left(2 \hat{\Pi}\left(D_{1} D_{2} U_{A}\right)+\Pi\left(D_{1} U_{A}\right)-2 \Pi\left(D_{2} U_{A}\right)\right), \\
& v_{D_{2}}=\frac{1}{6}\left(2 \hat{\Pi}\left(D_{1} D_{2} U_{A}\right)-2 \Pi\left(D_{1} U_{A}\right)+\Pi\left(D_{2} U_{A}\right)\right) .
\end{aligned}
$$

However, suppose that $U_{A}$ negotiated with $D_{1}$ followed by $D_{2}$, then by refusing to negotiate with $D_{1}$ and causing an eventual breakdown, $U_{A}$ would receive $\frac{1}{2} \Pi\left(D_{2} U_{A}\right)$ from an agreement with $D_{2}$ alone. If $\Pi\left(D_{2} U_{A}\right)-\frac{1}{2} \Pi\left(D_{1} U_{A}\right)>\hat{\Pi}\left(D_{1} D_{2} U_{A}\right)$, both $U_{A}$ and $D_{1}$ would prefer a breakdown to an agreement and hence, an equilibrium involving both downstream firms being active would not be possible. Observe that this preference would not occur in the absence of externalities; hence feasibility is not an issue in that case.

Importantly, note that, in contrast to other papers on competitive externalities such as Hart and Tirole (1990), Rey and Tirole (2003) and Chemla (2003), we allow the upstream firm to exclude one downstream firm or the other. What constrains the incentive to exclude, however, is that after triggering a breakdown the upstream firm would face only a single downstream firm, with greater bargaining power as a result. The upstream firm trades off competitive externalities against the loss in bargaining power.

For the remainder of this paper, we will assume that the feasibility conditions hold regardless of the level of integration. Nonetheless, in the appendix, we provide the conditions for feasibility to hold in our model (including our running example).

Given feasibility, we can demonstrate the following: 
Proposition 4. In a perfect Bayesian equilibrium with passive beliefs, each firm receives the payoffs listed in Table 2.

The payoffs in Table 2 are particularly interesting: they are not classical Shapley values. First, surplus is not maximized because payoffs reflect the presence of externalities. Second, the distribution of the surplus generated does not take a Shapley form. For example, payoffs are a function of $\hat{\Pi}\left(\overline{D_{1} U_{A}}, \overline{D_{2} U_{B}}\right)$, the profit earned by $U_{A}$ and $D_{I}$ jointly when $U_{A}$ supplies $D_{l}$, and they face competing supply in the downstream market from $D_{2}$, supplied by $U_{B}$. In contrast, Shapley values do not allow one's payoff to depend on the configuration of players that one is not cooperating with. ${ }^{45}$ In effect these payoffs are allowing for the effect of competitive externalities. Notice that when there are no externalities, the payoffs in Table 2 collapse to Shapley values (as in Table 1); that is, profits are maximized under all market structures and, say, $\hat{\Pi}\left(\overline{D_{1} U_{B}}, \overline{D_{2} U_{A}}\right)=\Pi\left(\overline{D_{1} U_{B}}\right)$.

Notice, however, that the Shapley value-type solution arises naturally in the upstream monopoly case. In that situation, $U_{B}$ can never produce independently of $U_{A}$, so the types of partitions that arise for the upstream competition case are ruled out. Thus, competitive externalities do not change the payoffs of each agent under upstream monopoly; save for the fact that industry profits are not maximized where both downstream firms are present.

\subsection{Comparing Incentives for FI and BI}

In Section 3, we asked whether the acquiring firm in vertical integration would come from the more or less competitive vertical segment. From Table 2, we can see that FI will be preferred to BI, under either upstream competition or monopoly, if and only if $\hat{\Pi}\left(\overline{D_{1} D_{2} U_{B}}\right) \geq \Pi\left(\overline{D_{2} U_{A} U_{B}}\right)$. This corresponds to the comparison made for the no externalities case except that here the left hand side takes into account the fact that when downstream outputs are substitutes in the eyes of consumers, industry profits will be lower as a result of their competition. Indeed, the more substitutable are downstream

${ }^{45}$ The payoffs here are related to cooperative games in partition function form (see Myerson (1980) for a discussion). The precise relationship of the payoffs here with cooperative game theory is an item for future research. 
outputs in the eyes of consumers (intensifying Cournot competition under nonintegration), the more likely it is that a downstream firm will acquire upstream assets. Hence, our conclusion that the acquirer will come from the more competitive vertical segment is strengthened when there are competitive externalities.

At this stage it is also instructive to consider the relative impacts of FI and BI on outsiders. Recall that, in general, analyses of the anticompetitive effects of vertical integration have not distinguished between the type of integration. While our analysis demonstrates that FI and BI have the same impact on total surplus, it also highlights their differential impact on bargaining positions; especially for outsiders.

The following table lists the benefits to outsiders from integration by $D_{1}$ and $U_{A}$ for a situation where upstream and downstream firms are symmetric and the conditions of Proposition 3 hold. In that case, FI always improves $D_{2}$ 's payoff, whether the acquirer of $D_{1}$ is an upstream competitor or monopolist. Essentially, any reduction in bargaining position is outweighed by the potential increase in industry profits. Thus, it is BI that would raise concerns for exit by $D_{2}$; indeed, when there are no competitive externalities, $D_{2}$ 's payoff is reduced by BI. In contrast, from upstream competition, FI reduces $U_{B}$ 's payoff; causing more concern regarding its potential exit than would be the case for BI. Thus, the FI-BI distinction can matter for antitrust analysis if potential exit of an outsider is an issue for evaluation.

\begin{tabular}{|c|c|c|}
\hline Integration Type & Benefit to $D_{2}$ & Benefit to $U_{B}$ \\
\hline UC-FI & $\frac{1}{\overline{12}}\left(\begin{array}{l}\Pi\left(\overline{D_{1} D_{2} U_{j}}\right)-2 \hat{\Pi}\left(\overline{D_{1} D_{2} U_{j}}\right) \\
+2 \Pi\left(\overline{D_{t} U_{j}}\right)\end{array}\right)>0$ & $\frac{1}{12}\left(2 \hat{\Pi}\left(\overline{D_{1} D_{2} U_{j}}\right)-3 \Pi\left(\overline{D_{1} D_{2} U_{j}}\right)\right)<0$ \\
\hline UC-BI & $\frac{1}{12}\left(\begin{array}{l}\Pi\left(\overline{D_{1} D_{2} U_{j}}\right)-\hat{\Pi}\left(\overline{D_{1} D_{2} U_{j}}\right) \\
-\left(\overline{D_{1} U_{A} U_{s}}\right)\end{array}\right)$ & $\frac{1}{12}\left(\begin{array}{l}3 \Pi\left(\overline{D_{1} D_{2} U_{j}}\right)-3 \Pi\left(\overline{D_{1} D_{2} U_{j}}\right) \\
-\Pi\left(\overline{D_{i} U_{A} U_{B}}\right)+2 \Pi\left(\overline{D_{i} U_{j}}\right)\end{array}\right)$ \\
\hline UM-FI & $\frac{1}{12}\left(\begin{array}{l}3 \Pi\left(\overline{D_{1} D_{2} U_{A} U_{B}}\right)-3 \hat{\Pi}\left(\overline{D_{1} D_{2} U_{A} U_{B}}\right) \\
+\Pi\left(\overline{D_{1} D_{2} U_{j}}\right)-\hat{\Pi}\left(\overline{D_{1} D_{2} U_{j}}\right)\end{array}\right)>0$ & $\frac{1}{12}\left(\begin{array}{l}3 \Pi\left(\overline{D_{1} D_{2} U_{A} U_{B}}\right)-3 \hat{\Pi}\left(\overline{D_{1} D_{2} U_{A} U_{B}}\right) \\
-3 \Pi\left(\overline{D_{1} D_{2} U_{j}}\right)+3 \hat{\Pi}\left(\overline{D_{1} D_{2} U_{j}}\right)\end{array}\right)$ \\
\hline UM-BI & $\left(\begin{array}{l}3 \Pi\left(\overline{D_{1} D_{2} U_{A} U_{B}}\right)-3 \hat{\Pi}\left(\overline{D_{1} D_{2} U_{A} U_{B}}\right) \\
+\Pi\left(\overline{D_{1} D_{2} U_{j}}\right) \\
-\Pi\left(\overline{D_{t} U_{A} U_{B}}\right)-2 \Pi\left(\overline{D_{i} U_{j}}\right)\end{array}\right)$ & 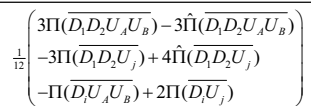 \\
\hline
\end{tabular}


The central question being considered in this paper is whether it is indeed the case that there is more incentive for vertical integration when there is upstream monopoly rather than upstream competition. When there are no competitive externalities, we concluded that due to pure bargaining effects, the greatest potential for purely strategic vertical integration arose under upstream competition rather than upstream monopoly.

When there are competitive externalities, vertical integration involves a monopolization effect and consequent welfare harm. In the special case of Proposition 3, this effect was stronger when there was a vertically integrated upstream monopolist rather than an upstream competitor. Nonetheless, using Table 2, we can compare the incentives for welfare-reducing vertical integration in each case.

Proposition 5. The increase in the joint payoff of $D_{I}$ and $U_{A}$ from both FI and BI under upstream competition will exceed that achieved under upstream monopoly if and only if $\frac{1}{3}\left(\hat{\Pi}\left(\overline{D_{1} D_{2} U_{B}}\right)-\Pi\left(\overline{D_{2} U_{B}}\right)\right) \geq \hat{\hat{\Pi}}_{U M}\left(\overline{D_{1} D_{2} U_{A} U_{B}}\right)-\hat{\hat{\Pi}}_{U C}\left(\overline{D_{1} D_{2} U_{A} U_{B}}\right)$.

The left hand side of the inequality in the proposition comes from the fact that the bargaining effect from vertical integration is stronger under upstream competition than monopoly. On the other hand, an upstream monopoly is able to use vertical integration more effectively to increase industry profits; thus, the monopolization effect weakens the relative incentives of an upstream competitor to vertically integrate.

Propositions 3 and 5 demonstrate that if downstream firms sell perfectly substitutable products, the conventional wisdom regarding the impact of upstream competition on the incentive to integrate is likely (although not guaranteed) to hold. In that case, the left hand side of the inequality in Proposition 5 is at its lowest while the right hand-side is at its highest possible level; as the upstream monopolist can achieve an industry monopoly outcome when it integrates while under upstream competition, integration leaves industry profits unchanged.

Nonetheless, as downstream products become less substitutable, it is likely that the reverse will be the case. Indeed, we know (from Section 3 ) that in the extreme where downstream firms operate in separate markets - there is a greater incentive to integrate under upstream competition. This suggests that as the degree of downstream 
product differentiation becomes sufficiently high, the conventional wisdom will be overturned. For our running example we can demonstrate that this is indeed the case.

EXAMPLE (Continued): Figure 5 illustrates the difference between the incentives for vertical integration (i.e., improvement to joint surplus relative to non-integration) under upstream competition less those under upstream monopoly. Note that the lower the degree of product differentiation, the lower is the relative incentive under upstream competition.

Figure 5: Differences in the Payoff Increase from Integration under Upstream Competition and Monopoly
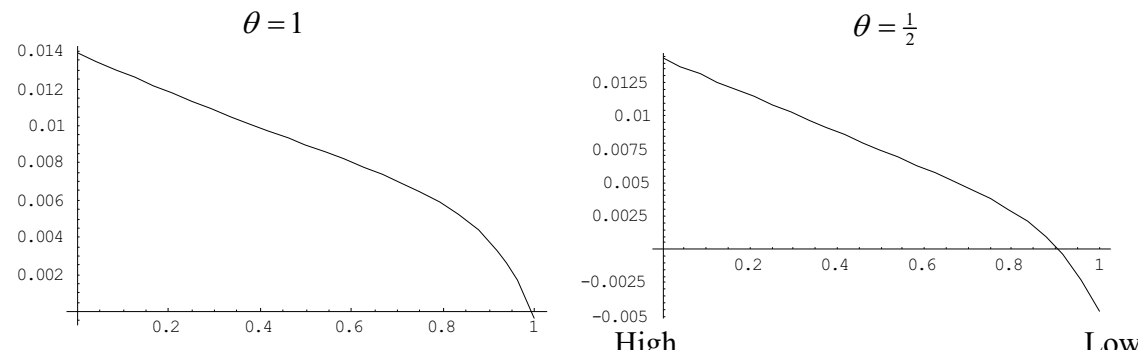

High Product Differentiation $(\gamma)$

It is worth emphasizing that the foreclosure effects of integration on nonintegrated firms differ in a subtle but important way from previous studies. An interesting feature of the upstream monopoly case is that, under the assumption of perfect symmetry and substitutability upstream and downstream, vertical integration leads to the monopoly output industry-wide. In that case, $D_{2}$ is not supplied any inputs and hence, does not produce, leaving $D_{1}$ to supply the monopoly quantity downstream. However, under FI, $D_{2}$ does receive a payoff of:

$$
v_{D_{2}}(F I)=\frac{1}{12}\left(\Pi\left(\overline{D_{1} D_{2} U_{A} U_{B}}\right)-\Pi\left(\overline{D_{i} U_{j}}\right)\right) \cdot{ }^{46}
$$

The reason for this is that even though $D_{2}$ plays no actual productive role, it does provide the integrated firm owner (in its internal negotiations under FI) an outside option in case 
of a bargaining breakdown with $D_{1} \cdot{ }^{47}$ Thus, while there is technical foreclosure in terms of the elimination of downstream competition, $U_{A}$ still cedes rents to $D_{2}$ so as to improve its bargaining position with respect to $D_{1}$ 's manager. ${ }^{48}$

\section{Counter-Mergers}

To date, our analysis has focused on consideration of the incentives and effects of a single vertical merger between $U_{A}$ and $D_{1}$. While this might be appropriate in situations where merger opportunities are limited (for instance, due to a need for technological or organizational compatibility or some other legal restraint), in other situations the possibility of a counter-merger remains and such mergers are observed (Chandler, 1964; Scherer and Ross, 1990).

Two related issues arise in this regard. First, are participants to a counter-merger reacting to the initial merger or would they have chosen this course independently? That is, is there a bandwagon effect associated with vertical integration? Second, does the possibility of a counter-merger alter the incentives for the initial merger? We analyze each of these questions in turn. As the possibility of a counter-merger is most salient when rival firms are similarly placed to the initial merging parties, we will assume throughout this section that upstream and downstream firms are symmetric. ${ }^{49}$

\footnotetext{
${ }^{47}$ A lump sum payment from upstream firms to $D_{2}$, without any corresponding input supply, might be seen as strange. The solution here can be approximated, however, by some arbitrarily small input supply to $D_{2}$. ${ }^{48}$ When upstream firms have constant costs (as in HT's 'Ex Post Monopolization' variant) but, say, $U_{4}$, costs are lower than $U_{B}$ 's, then $U_{B}$ does not supply either downstream firm under non-integration or integration. However, while in HT, this implies that $U_{B}$ receives no payoff, here that is only the case under upstream monopoly. Under upstream competition, so long as $U_{B}$ is not too inefficient, $U_{B}$ receives a upstream monopoly. Under upstream competition, so long as $U_{B}$ is not too inefficient, $U_{B}$ receives a
payment from $D_{1}$ (or the integrated firm) so as it improve its bargaining position in the event of an internal breakdown. However, it always receives a payment from $D_{2}$. Hence, even with FI, $U_{B}$ may not wish to exit the industry. This result is very similar to $\mathrm{SZ}$ who find that a firm will employ more workers than would be efficient in order to reduce the bargaining position of all its workers. Here, however, will an essentially inactive firm might receive a payment, it is not in the payer's interest to require that firm produce. In the SZ case, it remains in the interests of the firm to utilise all employed workers. If a pool of inactive, replacement workers was available this would change the SZ result (see de Fontenay and Gans, 2003a).

${ }_{49}$ This assumption is made for simplicity of exposition. We derive all proofs of results in this section in

Appendix $\mathrm{C}$ without this assumption.
} 
Here we explore whether an initial merger may encourage or discourage further mergers. One measure of this type of interrelationship is to compare the follow-on incentive for a merger (that is, the increase in joint payoff for, say, $U_{B}$ and $D_{2}$ if their merger follows that of $U_{A}$ and $D_{1}$ ) with their incentive for a stand-alone merger (presuming that $U_{A}$ and $D_{1}$ remain vertically separated). As will be demonstrated when we look at the full equilibrium below, if the follow-on incentive for a merger is greater than the stand-alone incentive, we can conclude that positive bandwagon effects exist; that is, an initial merger may precipitate further mergers in the industry. ${ }^{50}$

Suppose that following forward or backwards integration by $U_{A}$ and $D_{1}, U_{B}$ and $D_{2}$ integrate in the same fashion. ${ }^{51}$ The impact of counter-mergers is only of relevance in the upstream competition case and we concentrate on that here. The payoffs following a counter-merger are as in Table 3 where NI, PI and CI are the states of non-integration, partial integration by either pair and (complete) integration by both pairs, respectively. ${ }^{52}$ The stand-alone incentives for $U_{B}$ and $D_{2}$ to integrate (absent a similar merger by $U_{A}$ and $\left.D_{1}\right)$ are:

$$
S_{B 2} \equiv \frac{1}{2}\left(\hat{\Pi}_{P I}\left(\overline{D_{1} D_{2} U_{A} U_{B}}\right)-\hat{\Pi}_{N I}\left(\overline{D_{1} D_{2} U_{A} U_{B}}\right)\right)+\frac{1}{6} \omega(s)
$$

where

$$
\omega(B I)=\hat{\hat{\Pi}}_{P I}\left(\overline{D_{1} D_{2} U_{j}}\right)-\hat{\Pi}_{N I}\left(\overline{D_{1} D_{2} U_{j}}\right)+\Pi\left(\overline{D_{i} U_{A} U_{B}}\right)-\Pi\left(\overline{D_{i} U_{j}}\right)
$$

${ }^{50}$ The past literature is divided on whether bandwagon effects arise. Some researchers examining the possibility of vertical foreclosure have constructed models whereby vertical integration reduces incentives for further integration. For instance, Ordover, Saloner and Salop (1990) argue that initial integration is driven by competition for assets and the negative externality of integration on non-integrated firms, something not present for later integration choices. Choi and Yi (2000) and Chen (2001) similarly demonstrate that potential negative externalities motivating initial integration are not present for later integration as such integration may 're-symmetrize' competition and trigger a strong competitive response. In contrast, HT and McLaren (2000) provide models whereby initial integration raises the incentives for In contrast, HT and McLaren (2000) provide models whereby initial integration raises the incentives for
further vertical integration. In each case, vertical integration exacerbates potential 'hold-up' problems faced by non-integrated firms and may drive them to integrate. Our approach here does not consider an asset by non-integrated firms and may drive them to integrate. Our approach here does not cons
market effect nor rely on the potential for vertical integration to resolve hold-up problems.

${ }_{51}$ As before, we need to make an assumption as to what would happen if negotiations broke down between the downstream unit of one firm and the other integrated firm. Analogous to our earlier assumption, we assume that in this case, no negotiations between the two firms would be possible - that is, the downstream unit of the other firm would not be able to purchase inputs outside their firm.

${ }_{52}$ The payoffs in Table 3 are calculated using the same procedure as in Table 2 (as documented in the proof of Proposition 4)

${ }_{33}^{3}$ Using symmetry and the results in Table 2. 
and $\omega(F I)=\hat{\hat{\Pi}}_{P I}\left(\overline{D_{1} D_{2} U_{j}}\right)-\Pi\left(\overline{D_{i} U_{j}}\right)$.

In contrast, the incentive for a counter-merger (that is, the increase in the joint payoff to $U_{B}$ and $D_{2}$ from CI over PI by $U_{A}$ and $\left.D_{1}\right)$ is:

$$
F_{B 2} \equiv \frac{1}{2}\left(\hat{\hat{\Pi}}_{C I}\left(\overline{D_{1} D_{2} U_{A} U_{B}}\right)-\hat{\hat{\Pi}}_{P I}\left(\overline{D_{1} D_{2} U_{A} U_{B}}\right)\right)+\frac{1}{6} \omega(s)
$$

There is a positive bandwagon effect if $F_{B 2}>S_{B 2}$. Regardless of whether there is BI or FI, this will occur if:

$$
\hat{\Pi}_{C I}\left(D_{1} D_{2} U_{A} U_{B}\right)+\hat{\Pi}_{N I}\left(D_{1} D_{2} U_{A} U_{B}\right)>2 \hat{\Pi}_{P I}\left(D_{1} D_{2} U_{A} U_{B}\right)
$$

That is, if the marginal increase in industry profits from moving to CI from PI is higher than that to PI from NI. If the inequality in (5) is reversed, then there is a negative bandwagon effect and the incentives for a counter-merger are reduced by the occurrence of an initial merger.

Observe that this bandwagon effect collapses to 0 when there are no competitive externalities or the conditions of Proposition 3 hold. In these cases, $U_{B}$ and $D_{2}$ 's incentives to merge are unchanged by what $U_{A}$ and $D_{1}$ may have done. The reason is that, in this case, the only impact from vertical integration comes from bargaining effects. In particular, integration by $U_{B}$ and $D_{2}$ only rules out possible market structures that involve profits that are the same regardless of whether $U_{A}$ and $D_{1}$ are integrated or not. Thus, the return to integration does not depend on prior integration.

When there is an impact on total profits from integration, bandwagon effects are possible. However, it is possible that integration could reduce industry profits. In this case, an initial merger may reduce the incentives for a second merger.

EXAMPLE (Continued): In our running example, Figure 6) illustrates the size of the bandwagon effect. The graph assumes that downstream firms care about the source of inputs $(\theta=1)$. The bandwagon effect is negative as product differentiation is reduced so that a first merger reduces incentives for a second parallel one. 
Figure 6: Bandwagon Effect $\left(F_{B 2}-S_{B 2}\right)$

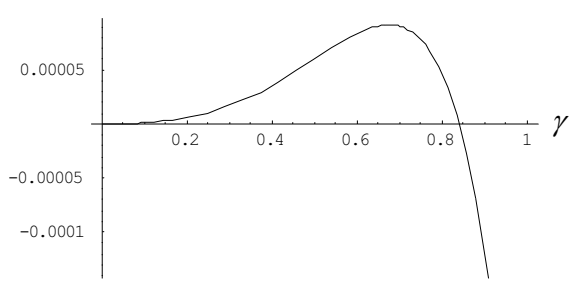

5.2 How does the possibility of a counter-merger impact on initial merger incentives?

Our analysis in Sections 3 and 4 has demonstrated that a merger between $U_{A}$ and $D_{1}$ can harm one or both of the remaining firms (at least insofar as bargaining effects are concerned). It is conceivable that $U_{B}$ and $D_{2}$ will have an opportunity to respond to the merger by themselves integrating; perhaps mitigating any bargaining advantage $U_{A}$ and $D_{1}$ were expecting to receive.

The effect of counter-mergers on any resulting 'asset market equilibrium' has received some attention in the literature on vertical foreclosure. HT and Ordover, Saloner and Salop (1990) (hereafter OSS) consider 'reduced form' merger games to evaluate whether an initial merger will still proceed if a counter-merger is possible. Here we consider the HT game (with a comparison to OSS relegated to Appendix C). ${ }^{54}$

HT assume that (i) $U_{A}$ can only merge with $D_{1}$ and $U_{B}$ with $D_{2} ;{ }^{55}$ (ii) integration is irreversible; and (iii) if one merger occurs the other pair can also follow suit prior to any bargaining or production. This last assumption is a critical one, allowing rival firms to respond quickly to a merger by others; thereby, raising the incentives for and potential deterrent effect of a counter-merger. Effectively, it corresponds to the extensive form game depicted in Figure 7. There, each pair simultaneously chooses whether to merge or not. If one pair does but the other does not, the latter has a further opportunity to merge

${ }^{54}$ Bolton and Whinston (1993) and Gans (2001) also consider market-based allocations of asset ownership. Those papers highlight the difficulties of providing general solutions given the nature of ownership externalities. For this reason we focus here to more specific merger games; illustrating the possible effects of counter-mergers rather than general asset market equilibrium.

${ }^{55}$ This is done without loss of generality given the symmetry assumption employed here. In Appendix C,

we analyse this game without imposing a symmetry assumption. 
but if neither chooses to merge, no further merger is possible. ${ }^{56}$

To see how this applies to our model, suppose that $U_{A}$ and $D_{1}$ always have a stand-alone incentive to integrate $\left(S_{A 1}>0\right)$. In this case, if $F_{B 2} \leq 0$, then there will be a subgame perfect equilibrium involving partial integration by $U_{A}$ and $D_{1} \cdot{ }^{57}$ Notice that, by symmetry, a necessary condition for this to be the case is that there are negative bandwagon effects (i.e., (5) does not hold). For this equilibrium, all of the analysis in Sections 3 and 4 holds even when a counter-merger is possible. Nonetheless, this equilibrium involving partial integration is ruled out if either there are no externalities or the conditions of Proposition 3 hold as $F_{B 2}=S_{B 2}>0$ in this case. In the no externality case, for example, integration results in pure rent distribution so that whenever there is a gain from an initial stand-alone merger, there must also be a similar offsetting gain from a counter-merger.

Both non-integration and complete integration (with two vertical mergers) are also possible equilibrium outcomes if $F_{A 1}, F_{B 2}>0$. In the CI equilibrium, both pairs merge initially and neither gains an advantage from (nor can commit to) not merging at all given the merger of their rivals. An NI equilibrium can co-exist with this one if pairs prefer NI to CI; that is, neither pair merges initially (and so there is no second period merger possibility) and each knows that if it does merge the other will counter-merge, resulting in CI.

Looking at the CI equilibrium, if $F_{B 2}>0$ (and by symmetry, $F_{A 1}>0$ ) then these become the relevant incentives for integration under upstream competition. Comparing this with the incentives for the integration of a single downstream firm under upstream monopoly, both BI and FI are more likely to occur under upstream competition if:

$$
\frac{1}{6}\left(\hat{\Pi}\left(\overline{D_{1} D_{2} U_{j}}\right)-\Pi\left(\overline{D_{i} U_{j}}\right)\right)>\frac{1}{2}\left(\hat{\hat{\Pi}}_{U M}\left(\overline{D_{1} D_{2} U_{A} U_{B}}\right)-\hat{\Pi}\left(\overline{D_{1} D_{2} U_{A} U_{B}}\right)-\hat{\hat{\Pi}}_{C I}\left(\overline{D_{1} D_{2} U_{A} U_{B}}\right)+\hat{\hat{\Pi}}_{P I}\left(\overline{D_{1} D_{2} U_{A} U_{B}}\right)\right)
$$

The LHS differs from the condition in Proposition 5. It is easy to see that the LHS of (6) is lower if (5) holds; that is, if there are positive bandwagon effects. In this case, the incentives to integrate under upstream competition continue to be greater than those

\footnotetext{
${ }^{56} \mathrm{HT}$ argue that this reduced form game corresponds generally to the outcomes of a more elaborate game in continuous time where in each period, each pair can choose whether to merge or not.

${ }^{57}$ This is demonstrated in Appendix C. Notice that $U_{B}$ and $D_{2}$ do not have an incentive to merge at the same time as the rival pair as their joint payoff under PI exceeds that under CI.
} 
under upstream monopoly. Notice that under the conditions of Proposition 3 or if there are no competitive externalities, then both conditions are equivalent and the incentive for $\mathrm{BI}$ and FI under a scenario leading to complete integration is no different from the standalone merger incentives.

In summary, our previous analysis of the relative incentives for integration from upstream competition as opposed to monopoly are generally robust to the inclusion of the possibility of a counter-merger. In terms of welfare effects, CI tends to lead to higher prices and lower consumer surplus than PI or NI, although higher consumer surplus than integration in upstream monopoly.

EXAMPLE (Continued): In our running example, Figure 8 adds CI to the welfare comparisons in Figure 4. Note that CI leads to lower industry profits and consumer surplus than PI or NI. Basically, CI distorts productive efficiency even further than PI: lowering industry profits overall.

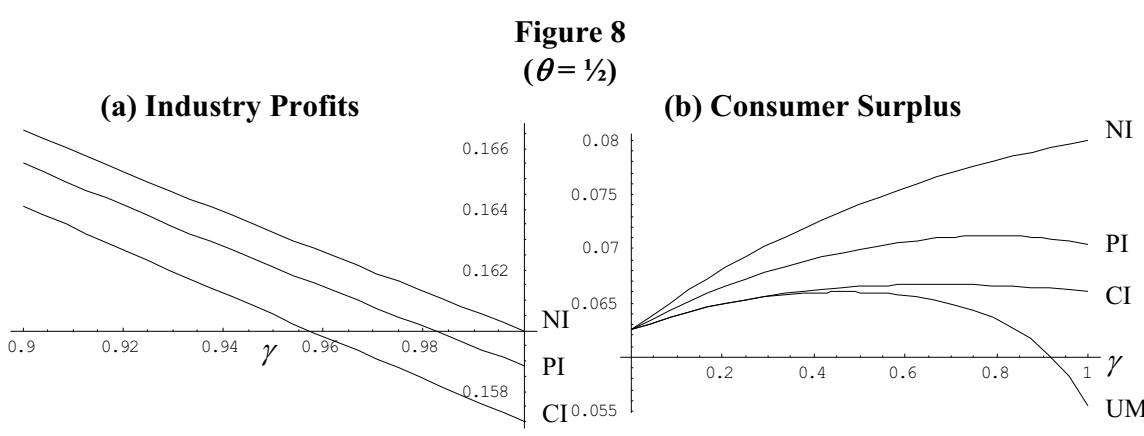

\section{Conclusions}

This paper has sorted out alternative claims regarding the impact of upstream competition on the incentives and consequences for vertical integration. While vertical integration that occurs when there is an upstream monopoly has the greatest potential to cause higher prices and lower consumer welfare, this need not translate into greater incentives for purely strategic vertical integration. Specifically, those incentives may be higher when there is upstream competition (especially if downstream competition is not too intense) and may be higher for backward integration (from the competitive into the monopolistic segment) than for forward integration (akin to the more conventional 
picture of an acquiring monopolistic firm).

In terms of competition and anti-trust analysis, our results support the notion that proposed vertical mergers involving a monopoly bottleneck are of greater concern than where there is upstream competition. Nonetheless, in terms of policies designed to restructure industries and encourage upstream competition (such as those that have occurred in cable television and telecommunications), the potential gains associated with these moves may be mitigated as it could encourage greater strategic vertical integration.

Nonetheless, while our model has synthesized and generalized existing models in the strategic vertical integration literature - as well as providing a framework linking these to models in the property rights literature - there are many possible extensions. In particular, moving beyond the simple 2 by 2 case would be useful. This could be by expanding the number of upstream and downstream assets as well as deepening the vertical chain of production. This would allow a mapping between our work and the work of Hendricks and McAfee (2000) who provide a means of linking concentration measures and integration in vertical segments with the potential for anticompetitive harm from a merger. Their work is based on a mechanism design approach to vertical relations whereas ours uses a non-cooperative bargaining model. This would also provide a means of dealing more carefully with the impact of vertical integration on entry. 


\section{Appendix A: Proofs of Propositions}

\section{Proof of Propositions 1, 2 and 4:}

The key to the proof lies in several steps. First, we demonstrate that the solution to our extensive form game are equations of the form specified by the bilateral Nash bargaining solution, for each pair, in each subgame of a given supply configuration. Second, we demonstrate that, given this, the surplus generated in each subgame is to maximise industry profits (if there are no competitive externalities in that subgame) or the Cournot outcome (if there are competitive externalities). Finally, we demonstrate that the realized payoffs are as listed in Tables 1 and 2 .

Step 1 (Bilateral Bargaining Outcomes): We wish to demonstrate that, say, $\left(\tilde{p}_{1 A}, \tilde{q}_{1 A}\right)$ satisfy:

$$
\tilde{q}_{1 A} \in \arg \max _{q_{1 A}} \pi_{1}\left(q_{1 A}, q_{1 B}, q_{2 A}, q_{2 B}\right)-c_{A}\left(q_{1 A}, q_{2 A}\right)
$$

and $\tilde{p}_{1 A} \in\left\{p_{1 A} \mid \pi_{1}\left(\tilde{q}_{1 A}, q_{1 B}, q_{2 A}, q_{2 B}\right)-p_{1 A}-p_{1 B}-\Phi_{1 A}=p_{1 A}+p_{2 A}-c_{A}\left(\tilde{q}_{1 A}, q_{2 A}\right)-\Phi_{A 1}\right\}$

where $\Phi_{14}$ and $\Phi_{A 1}$ represent the payoffs $D_{1}$ and $U_{A}$ expect to receive in the renegotiation subgame triggered by a breakdown in their negotiations. Pairwise bargaining takes an alternating offer format. To fix ideas, suppose that $D_{i}$ makes the first offer that $U_{j}$ chooses to accept or reject. If the offer is accepted, the negotiation ends on the basis of that offer and the game moves on to the next negotiating pair or ends as the case may be. If it is rejected, then with probability $(1-\sigma)$ the negotiation ends with no supply taking place between the pair and the game moves on to the next negotiating pair. With probability $\sigma$, however, $U_{j}$ is able to make a counter-offer that may be accepted or rejected on the same basis as $D_{i}$ 's original offer. Offers alternate until one is accepted or there is an exogenous breakdown in negotiations. This format is the same as Binmore, Rubinstein and Wolinsky (1986) for bilateral negotiations. The subtlety here comes from the potential interrelationships between negotiations in a given sequence.

Let $\left(\tilde{p}_{i j}, \tilde{q}_{i j}\right)$ be the actual outcomes of negotiations between $D_{i}$ and $U_{j}$ and $\left(\hat{p}_{i j}, \hat{q}_{i j}\right)$ be the beliefs of downstream firms other than $i$ and upstream firms other than $j$ about the outcomes of those negotiations. Clearly for firms that were part of a particular negotiation, $\left(\hat{p}_{i j}, \hat{q}_{i j}\right)=\left(\tilde{p}_{i j}, \tilde{q}_{i j}\right)$. Otherwise, we assume that the agents hold passive beliefs.

Rey and Verge (2003) provide a definition of passive beliefs that we rely upon here. Here is a definition in relation to a given downstream firm, $i$. The converse definition for beliefs of upstream firms is analogous. 
Definition (Passive Beliefs). Let $\left(\hat{p}_{i j}, \hat{q}_{i j}\right)$ be $i$ and $j$ 's beliefs about the offer it expects to receive from $j$ and let $\left(\hat{p}_{k j}, \hat{q}_{k j}\right)$ be $i$ 's beliefs about the agreements $j$ will make with any other firm, $k \neq i$. When i receives an offer from $j$ of $\left(p_{i j}, q_{i j}\right) \neq\left(\hat{p}_{i j}, \hat{q}_{i j}\right)$, it believes that:

1. $j$ expects it to accept this offer,

2. $\left(\hat{p}_{k j}, \hat{q}_{k j}\right)$ will not change,

3. $k$ reasons the same way.

Suppose first that $U_{A}$ and $D_{1}$ are the last pair negotiating. One possible equilibrium outcome has $U_{A}$ and $D_{1}$ accepting offers made to them. This requires that when $D_{1}$ makes an offer it solves:

$$
\begin{aligned}
& \max _{\left(p_{1 A}, q_{1 A}\right)} \pi_{1}\left(q_{1 A}, \tilde{q}_{1 B}, \hat{q}_{2 A}, \hat{q}_{2 B}\right)-p_{1 A}-\tilde{p}_{1 B} \\
& \text { s.t. } p_{1 A}+\hat{p}_{2 A}-c_{A}\left(q_{1 A}, \hat{q}_{2 A}\right) \geq \sigma \hat{V}_{U_{A}}+(1-\sigma) \Phi_{A 1}
\end{aligned}
$$

where $\hat{V}_{U_{A}}$ is $D_{1}$ 's beliefs about $U_{A}$ 's expected payoff from an agreement (i.e., the solution to (UA) below). That is, it maximizes its payoffs subject to $U_{A}$ accepting its offer. $D_{1}$ will choose $p_{1 A}$ so that $U_{A}$ 's participation constraint binds. Notice that $U_{A}$ will only accept this offer if:

$$
\tilde{p}_{2 A}-c_{A}\left(q_{1 A}, \tilde{q}_{2 A}\right)-\sigma \tilde{V}_{U_{A}} \geq \hat{p}_{2 A}-c_{A}\left(q_{1 A}, \hat{q}_{2 A}\right)-\sigma \hat{V}_{U_{A}}
$$

being $U_{A}$ 's equilibrium participation constraint where $\tilde{V}_{U_{A}}$ is $U_{A}$ 's expected payoff from an agreement. beliefs):

In contrast, if $U_{A}$ makes an offer it solves (where it knows that $D_{1}$ holds passive

$$
\max _{\left(p_{1 A}, q_{1 A}\right)} p_{1 A}+\tilde{p}_{2 A}-c_{A}\left(q_{1 A}, \tilde{q}_{2 A}\right)
$$

s.t. $\pi_{1}\left(q_{1 A}, \hat{q}_{1 B}, \hat{q}_{2 A}, \hat{q}_{2 B}\right)-p_{1 A}-\hat{p}_{1 B} \geq \sigma \hat{V}_{D_{1}}+(1-\sigma) \Phi_{1 A}$

However, $D_{1}$ will only accept this offer if:

$$
\pi_{1}\left(q_{1 A}, \tilde{q}_{1 B}, \hat{q}_{2 A}, \hat{q}_{2 B}\right)-\tilde{p}_{1 B}-\sigma \tilde{V}_{D_{1}} \geq \pi_{1}\left(q_{1 A}, \hat{q}_{1 B}, \hat{q}_{2 A}, \hat{q}_{2 B}\right)-\hat{p}_{1 B}-\sigma \hat{V}_{D_{1}} \quad \text { (PC-D1) }
$$

Notice that the maximisation problems - (D1) and (UA) - imply that:

$$
\begin{aligned}
& \tilde{V}_{D_{1}}=\max _{q_{1 A}} \pi_{1}\left(q_{1 A}, \tilde{q}_{1 B}, \hat{q}_{2 A}, \hat{q}_{2 B}\right)-\tilde{p}_{1 B}+\hat{p}_{2 A}-c_{A}\left(q_{1 A}, \hat{q}_{2 A}\right)-\sigma \hat{V}_{U_{A}}-(1-\sigma) \Phi_{A 1} \\
& \tilde{V}_{U_{A}}=\max _{q_{1 A}} \pi_{1}\left(q_{1 A}, \hat{q}_{1 B}, \hat{q}_{2 A}, \hat{q}_{2 B}\right)-\hat{p}_{1 B}+\tilde{p}_{2 A}-c_{A}\left(q_{1 A}, \tilde{q}_{2 A}\right)-\sigma \hat{V}_{D_{1}}-(1-\sigma) \Phi_{1 A} \\
& \hat{V}_{D_{1}}=\max _{q_{1 A}} \pi_{1}\left(q_{1 A}, \hat{q}_{1 B}, \hat{q}_{2 A}, \hat{q}_{2 B}\right)-\hat{p}_{1 B}+\hat{p}_{2 A}-c_{A}\left(q_{1 A}, \hat{q}_{2 A}\right)-\sigma \hat{V}_{U_{A}}-(1-\sigma) \Phi_{A 1} \\
& \hat{V}_{U_{A}}=\max _{q_{1 A}} \pi_{1}\left(q_{1 A}, \hat{q}_{1 B}, \hat{q}_{2 A}, \hat{q}_{2 B}\right)-\hat{p}_{1 B}+\hat{p}_{2 A}-c_{A}\left(q_{1 A}, \hat{q}_{2 A}\right)-\sigma \hat{V}_{D_{1}}-(1-\sigma) \Phi_{1 A}
\end{aligned}
$$

There are four equations and four unknowns. Notice that the last two involve the same quantity choice (let this be $\hat{q}_{1 A}$ ) while we denote the quantity choice in the first and second as $\tilde{q}_{1 A}^{1}$ and $\tilde{q}_{1 A}^{A}$, respectively. Solving the last two yields:

$$
\begin{aligned}
& \hat{V}_{D_{1}}=\frac{1}{1+\sigma}\left(\pi_{1}\left(\hat{q}_{1 A}, \hat{q}_{1 B}, \hat{q}_{2 A}, \hat{q}_{2 B}\right)-\hat{p}_{1 B}+\hat{p}_{2 A}-c_{A}\left(\hat{q}_{1 A}, \hat{q}_{2 A}\right)+\sigma \Phi_{1 A}-\Phi_{A 1}\right) \\
& \hat{V}_{U_{A}}=\frac{1}{1+\sigma}\left(\pi_{1}\left(\hat{q}_{1 A}, \hat{q}_{1 B}, \hat{q}_{2 A}, \hat{q}_{2 B}\right)-\hat{p}_{1 B}+\hat{p}_{2 A}-c_{A}\left(\hat{q}_{1 A}, \hat{q}_{2 A}\right)-\Phi_{1 A}+\sigma \Phi_{A 1}\right) .
\end{aligned}
$$


Notice that these correspond to the payoff outcomes that would result from the conjectured bilateral bargaining outcome.

$$
\begin{aligned}
& \text { Given this (PC-UA) becomes: } \\
& (1-\sigma) \tilde{p}_{2 A}-c_{A}\left(\tilde{q}_{1 A}^{1}, \tilde{q}_{2 A}\right)-\sigma\left(\pi_{1}\left(\tilde{q}_{1 A}^{A}, \hat{q}_{1 B}, \hat{q}_{2 A}, \hat{q}_{2 B}\right)-c_{A}\left(\tilde{q}_{1 A}^{A}, \tilde{q}_{2 A}\right)\right) \\
& \geq(1-\sigma) \hat{p}_{2 A}-c_{A}\left(\tilde{q}_{1 A}^{1}, \hat{q}_{2 A}\right)-\sigma\left(\pi_{1}\left(\hat{q}_{1 A}, \hat{q}_{1 B}, \hat{q}_{2 A}, \hat{q}_{2 B}\right)-c_{A}\left(\hat{q}_{1 A}, \hat{q}_{2 A}\right)\right)
\end{aligned}
$$

and (PC-D1) becomes:

$\pi_{1}\left(\tilde{q}_{1 A}^{A}, \tilde{q}_{1 B}, \hat{q}_{2 A}, \hat{q}_{2 B}\right)-\tilde{p}_{1 B}(1-\sigma)-\sigma\left(\pi_{1}\left(\tilde{q}_{1 A}^{1}, \tilde{q}_{1 B}, \hat{q}_{2 A}, \hat{q}_{2 B}\right)-c_{A}\left(\tilde{q}_{1 A}^{1}, \hat{q}_{2 A}\right)\right)$

$\geq \pi_{1}\left(\tilde{q}_{1 A}^{A}, \hat{q}_{1 B}, \hat{q}_{2 A}, \hat{q}_{2 B}\right)-\hat{p}_{1 B}(1-\sigma)-\sigma\left(\pi_{1}\left(\hat{q}_{1 A}, \hat{q}_{1 B}, \hat{q}_{2 A}, \hat{q}_{2 B}\right)-c_{A}\left(\hat{q}_{1 A}, \hat{q}_{2 A}\right)\right)$

Re-arranging these constraints and taking the limit as $\sigma$ approaches 1 , it is easy to see that both inequalities will hold only if:

$$
\begin{aligned}
& \max \left[c_{A}\left(\tilde{q}_{1 A}^{1}, \tilde{q}_{2 A}\right)-c_{A}\left(\tilde{q}_{1 A}^{A}, \tilde{q}_{2 A}\right), \pi_{1}\left(\tilde{q}_{1 A}^{1}, \tilde{q}_{1 B}, \hat{q}_{2 A}, \hat{q}_{2 B}\right)-\pi_{1}\left(\tilde{q}_{1 A}^{A}, \tilde{q}_{1 B}, \hat{q}_{2 A}, \hat{q}_{2 B}\right)\right] \\
& \leq \pi_{1}\left(\hat{q}_{1 A}, \hat{q}_{1 B}, \hat{q}_{2 A}, \hat{q}_{2 B}\right)-c_{A}\left(\hat{q}_{1 A}, \hat{q}_{2 A}\right)-\pi_{1}\left(\tilde{q}_{1 A}^{A}, \hat{q}_{1 B}, \hat{q}_{2 A}, \hat{q}_{2 B}\right)+c_{A}\left(\tilde{q}_{1 A}^{1}, \hat{q}_{2 A}\right)
\end{aligned}
$$

Notice that if $\tilde{q}_{1 A}^{1}=\tilde{q}_{1 A}^{A}=\hat{q}_{1 A}$ this holds with equality. This will occur if $\tilde{q}_{2 A}=\hat{q}_{2 A}$ and $\tilde{q}_{1 B}=\hat{q}_{1 B}$. Thus, the conjectured equilibrium for this negotiation is, in fact, an equilibrium outcome where any offer by any party is immediately accepted; that is, the parties receive $\hat{V}_{D_{1}}$ and $\hat{V}_{U_{A}}$, respectively. In this situation, the above bilateral bargaining outcomes will emerge, so long as the conjectured equilibrium outcomes result from all other bilateral negotiations.

We now consider whether there might be a deviation in an earlier negotiation. If there is such a deviation what does the equilibrium of the bilateral bargaining subgame become? Recall that:

$$
\begin{aligned}
& \tilde{q}_{1 A}^{1}=\arg \max _{q_{1 A}} \pi_{1}\left(q_{1 A}, \tilde{q}_{1 B}, \hat{q}_{2 A}, \hat{q}_{2 B}\right)-c_{A}\left(q_{1 A}, \hat{q}_{2 A}\right) \\
& \tilde{q}_{1 A}^{A}=\arg \max _{q_{1 A}} \pi_{1}\left(q_{1 A}, \hat{q}_{1 B}, \hat{q}_{2 A}, \hat{q}_{2 B}\right)-c_{A}\left(q_{1 A}, \tilde{q}_{2 A}\right)
\end{aligned}
$$

Thus, if $\tilde{q}_{2 A}=\hat{q}_{2 A}$ but $\tilde{q}_{1 B} \neq \hat{q}_{1 B}$, then $\tilde{q}_{1 A}^{A}=\hat{q}_{1 A}$ and (PC-UA)' holds with equality (as $\sigma$ goes to 1 ); so $U_{A}$ will accept $D_{1}$ 's offer. However, (PC-D1)' becomes: $\pi_{1}\left(\hat{q}_{1 A}, \tilde{q}_{1 B}, \hat{q}_{2 A}, \hat{q}_{2 B}\right)-c_{A}\left(\hat{q}_{1 A}, \hat{q}_{2 A}\right) \geq \pi_{1}\left(\tilde{q}_{1 A}^{1}, \tilde{q}_{1 B}, \hat{q}_{2 A}, \hat{q}_{2 B}\right)-c_{A}\left(\tilde{q}_{1 A}^{1}, \hat{q}_{2 A}\right)$

which cannot be true by the definition of $\tilde{q}_{1 A}^{1}$. Similarly, if $\tilde{q}_{1 B}=\hat{q}_{1 B}$ but $\tilde{q}_{2 A} \neq \hat{q}_{2 A}$, then $\tilde{q}_{1 A}^{1}=\hat{q}_{1 A}$ and (PC-D1)' holds with equality; so $D_{1}$ will accept $U_{A}$ 's offer. However, (PCUA)' cannot hold by the definition of $\tilde{q}_{1 A}^{A}$, so $U_{A}$ will reject $D_{1}$ 's offer. Thus, an earlier deviation by a negotiating pair leads to an equilibrium in the bargaining game whereby one party accepts an offer while the other rejects and waits to make a counter-offer. The rejecting party is the party that was a party to an earlier deviation.

The question now becomes: anticipating this equilibrium outcome will an earlier deviation occur? Consider a deviation by $D_{1}$ and $U_{B}$. This results in $D_{1}$ 's offer of $\tilde{p}_{1 A}=-\hat{p}_{2 A}+c_{A}\left(\tilde{q}_{1 A}^{1}, \hat{q}_{2 A}\right)+\sigma \hat{V}_{U_{A}}+(1-\sigma) \Phi_{A 1}$ being accepted by $U_{A}$. Because of passive beliefs, $U_{B}$ will not deviate in its offers to $D_{1}$ as it perceives that this will not impact on the later negotiation between $D_{1}$ and $U_{A}$. In contrast, when $D_{1}$ makes an offer it anticipates the impact on the later negotiation when it solves: 
$\max _{q_{1 B}} \pi_{1}\left(\tilde{q}_{1 A}^{1}\left(q_{1 B}\right), q_{1 B}, \hat{q}_{2 A}, \hat{q}_{2 B}\right)-(\underbrace{-\hat{p}_{2 A}+c_{A}\left(\tilde{q}_{1 A}^{1}\left(q_{1 B}\right), \hat{q}_{2 A}\right)+\sigma \hat{V}_{U_{A}}+(1-\sigma) \Phi_{A 1}}_{=\tilde{p}_{1 A}})+\hat{p}_{2 B}-c_{B}\left(q_{1 B}, \hat{q}_{2 B}\right)$.

Notice that the impact on $\tilde{q}_{1 A}^{1}$ is of second order, by the envelope theorem, and so the solution to this problem is $\hat{q}_{1 B}$ (that is, there is no deviation from the conjectured equilibrium).

Now consider a deviation by $D_{2}$ and $U_{A}$. Substituting in $U_{A}$ 's offer to $D_{1}, U_{A}$ will make an offer to $D_{2}$ that solves (assuming downstream firms make the first offer in any negotiation and $D_{2}$ continues to holds passive beliefs, $\left.\hat{q}_{1 A}\right)$ :

$$
\max _{q_{2 A}} \pi_{2}\left(\hat{q}_{1 A}, \hat{q}_{1 B}, q_{2 A}, \hat{q}_{2 B}\right)-\hat{p}_{2 B}+\sigma(\underbrace{\pi_{1}\left(\tilde{q}_{1 A}^{A}\left(q_{2 A}\right), \hat{q}_{1 B}, \hat{q}_{2 A}, \hat{q}_{2 B}\right)-\hat{p}_{1 B}-\sigma \hat{V}_{D_{1}}-(1-\sigma) \Phi_{1 A}})-c_{A}\left(\tilde{q}_{1 A}^{A}\left(q_{2 A}\right), q_{2 A}\right)
$$

Note that it is not necessary to consider the offer from $D_{2}$ as its beliefs have not changed. The first order condition involves:

$$
\frac{\partial \pi_{2}\left(\hat{q}_{1 A}, \hat{q}_{1 B}, q_{2 A}, \hat{q}_{2 B}\right)}{\partial q_{2 A}}-\frac{\partial c_{A}\left(\tilde{q}_{1 A}^{A}\left(q_{2 A}\right), q_{2 A}\right)}{\partial q_{2 A}}+\underbrace{\frac{d \tilde{q}_{1 A}^{A}\left(q_{2 A}\right)}{d q_{2 A}}}_{0}\left(\frac{\partial \pi_{1}\left(\tilde{q}_{1 A}^{A}\left(q_{2 A}\right), \hat{q}_{1 B}, \hat{q}_{2 A}, \hat{q}_{2 B}\right)}{\partial q_{1 A}}-\frac{\partial c_{A}\left(\tilde{q}_{1 A}^{A}\left(q_{2 A}\right), q_{2 A}\right)}{\partial q_{1 A}}\right)=0 .
$$

Evaluate this at $q_{2 A}=\hat{q}_{2 A}$ (as defined by the equivalent condition to (7)) gives:

$$
\underbrace{\frac{\partial \pi_{2}\left(\hat{q}_{1 A}, \hat{q}_{1 B}, \hat{q}_{2 A}, \hat{q}_{2 B}\right)}{\partial q_{2 A}}-\frac{\partial c_{A}\left(\tilde{q}_{1 A}^{A}\left(q_{2 A}\right), \hat{q}_{2 A}\right)}{\partial q_{2 A}}}_{=0}+\underbrace{\frac{d \tilde{q}_{1 A}^{A}\left(q_{2 A}\right)}{d q_{2 A}}}_{<0}(\underbrace{\frac{\partial \pi_{1}\left(\hat{q}_{1 A}, \hat{q}_{1 B}, \hat{q}_{2 A}, \hat{q}_{2 B}\right)}{\partial q_{1 A}}-\frac{\partial c_{A}\left(\hat{q}_{1 A}, \hat{q}_{2 A}\right)}{\partial q_{1 A}}}_{=0})=0 .
$$

Thus, even anticipating the outcomes from a deviation, $U_{A}$ does not find it profitable to deviate from the proposed equilibrium.

It is easy to see that the logic used here did not rely on $U_{A}$ and $D_{1}$ 's actual place in the sequence of negotiations. Hence, under passive beliefs, the Binmore, Rubinstein, Wolinsky (1986) outcomes for bilateral negotiations continues to hold in this case. Moreover, the situation where there are four supply negotiations can be readily derived for the three and two negotiation case.

Step 2 (Surplus): The above analysis demonstrates that in an individual bilateral negotiation, quantity will be chosen to maximize the joint profits of each negotiating pair, taking the outcomes of other negotiations as given. That is, $q_{i j}^{*} \in \arg \max _{q_{i j}} \pi_{i}\left(q_{i A}, q_{i B}, q_{-i A}, q_{-i B}\right)-c_{j}\left(q_{1 j}, q_{2 j}\right)$. If there are no competitive externalities downstream and quantities can be renegotiated in any breakdown subgame, under passive beliefs, these are the only terms in industry profits containing $q_{i j}$; hence, if all negotiating pairs choose their respective quantities to maximize joint profits, by our concavity assumptions, industry profits will be maximized. This establishes efficiency for the no externality case (Proposition 1).

When there are competitive externalities, each pair chooses a quantity that maximizes joint profits taking the quantities chosen in other pairs as given. However, these quantities are chosen in a manner that equates marginal downstream profit to marginal upstream cost. Note that if instead downstream firms chose their quantities based on a per unit upstream price, say $\rho_{i j}$, they would choose their quantities to satisfy $\frac{\partial \pi_{i}}{\partial q_{i j}}=\rho_{i j}$. If $\rho_{i j}=\frac{\partial c_{j}}{\partial q_{i j}}$, then this will yield the same outcome as in each negotiation 
(establishing Proposition 2).

Under integration the quantities change for negotiations between $D_{2}$ and $U_{A}$. In this case, maximizing bilateral surplus is equivalent to: $\max _{q_{2 B}} \pi_{2}\left(q_{1 A}, q_{1 B}, q_{2 A}, q_{2 B}\right)+\pi_{1}\left(q_{1 A}, q_{1 B}, q_{2 A}, q_{2 B}\right)-c_{A}\left(q_{1 A}, q_{2 A}\right)$. The form of the quantity choice problems in the other negotiations will be unchanged. However, the change in one negotiation may lead to different quantities in equilibrium.

Step 3 (Distribution): For distribution, given passive beliefs, in the initial subgame, there are four bargaining pairs, the pricing outcomes of which are described by the following equations (as $\sigma$ goes to 1 ).

$$
\begin{aligned}
& \pi_{1}\left(q_{1 A}, q_{1 B}, q_{2 A}, q_{2 B}\right)-\tilde{p}_{1 A}-\tilde{p}_{1 B}-\Phi_{1 A}=\tilde{p}_{1 A}+\tilde{p}_{2 A}-c_{A}\left(q_{1 A}, q_{2 A}\right)-\Phi_{A 1} \\
& \pi_{1}\left(q_{1 A}, q_{1 B}, q_{2 A}, q_{2 B}\right)-\tilde{p}_{1 A}-\tilde{p}_{1 B}-\Phi_{1 B}=\tilde{p}_{1 B}+\tilde{p}_{2 B}-c_{B}\left(q_{1 B}, q_{2 B}\right)-\Phi_{B 1} \\
& \pi_{2}\left(q_{1 A}, q_{1 B}, q_{2 A}, q_{2 B}\right)-\tilde{p}_{2 A}-\tilde{p}_{2 B}-\Phi_{2 A}=\tilde{p}_{1 A}+\tilde{p}_{2 A}-c_{A}\left(q_{1 A}, q_{2 A}\right)-\Phi_{A 2} \\
& \pi_{2}\left(q_{1 A}, q_{1 B}, q_{2 A}, q_{2 B}\right)-\tilde{p}_{2 A}-\tilde{p}_{2 B}-\Phi_{2 B}=\tilde{p}_{1 B}+\tilde{p}_{2 B}-c_{B}\left(q_{1 B}, q_{2 B}\right)-\Phi_{B 2}
\end{aligned}
$$

where $\Phi_{i j}$ and $\Phi_{j i}$ represent the payoffs $D_{i}$ and $U_{j}$ expect to receive in the renegotiation subgame triggered by a breakdown in their negotiations. Solving these equations recursively, including the payoffs of each renegotiation subgame (that is, 88 simultaneous pricing equations), allows us to derive the equilibrium payoffs of each firm as in Table 2 (Proposition 4).

Under integration, the equations change. For example, for FI, the resulting (Nash) bargaining equations for price become:

$$
\tilde{t}_{1 A}-\Phi_{1 A}=\pi_{1}\left(q_{1 A}, q_{1 B}, q_{2 A}, q_{2 B}\right)-\tilde{t}_{1 A}-\tilde{p}_{1 B}+\tilde{p}_{2 A}-c_{A}\left(q_{1 A}, q_{2 A}\right)-\Phi_{A 1}
$$

$\pi_{1}\left(q_{1 A}, q_{1 B}, q_{2 A}, q_{2 B}\right)-\tilde{t}_{1 A}-\tilde{p}_{1 B}+\tilde{p}_{2 A}-c_{A}\left(q_{1 A}, q_{2 A}\right)-\Phi_{A B}=\tilde{p}_{1 B}+\tilde{p}_{2 B}-c_{B}\left(q_{1 B}, q_{2 B}\right)-\Phi_{B A}(13)$

$\pi_{2}\left(q_{1 A}, q_{1 B}, q_{2 A}, q_{2 B}\right)-\tilde{p}_{2 A}-\tilde{p}_{2 B}-\Phi_{2 A}=\pi_{1}\left(q_{1 A}, q_{1 B}, q_{2 A}, q_{2 B}\right)-\tilde{t}_{1 A}-\tilde{p}_{1 B}+\tilde{p}_{2 A}-c_{A}\left(q_{1 A}, q_{2 A}\right)-\Phi_{A 2}(14)$

$\pi_{2}\left(q_{1 A}, q_{1 B}, q_{2 A}, q_{2 B}\right)-\tilde{p}_{2 A}-\tilde{p}_{2 B}-\Phi_{2 B}=\tilde{p}_{1 B}+\tilde{p}_{2 B}-c_{B}\left(q_{1 B}, q_{2 B}\right)-\Phi_{B 2}$

Notice that there is a change in negotiating pairs relative to the non-integrated case. $U_{A}$ negotiates a supply agreement with $U_{B}$ for the supply of inputs to $D_{1}$. This is because the residual control rights of the downstream asset have been transferred to $U_{A}$. Again, solving these equations recursively, including the payoffs of each renegotiation subgame, allows us to derive the equilibrium payoffs of each firm as in Table 2 (Proposition 4).

Contingent Contracts: Finally, we demonstrate that the above equilibrium is also an equilibrium when pairs can negotiate contracts contingent upon the breakdown of others Suppose that $U_{A}$ and $D_{1}$ were the first pair to negotiate and consider a situation where they expect other pairs to reach agreement so long as they themselves continue to reach agreement. Let $\left(\tilde{p}_{1 A}(m), \tilde{q}_{1 A}(m)\right)$ be the price and quantity pairs between $U_{A}$ and $D_{1}$ contingent upon market structure, $m$. In this case, $U_{A}$ and $D_{1}$ will choose quantities to maximise their bilateral surplus when $m=D_{1} D_{2} U_{A} U_{B}$. Moreover, under passive beliefs, for other market structures, any deviation from the equilibrium where they maximise their bilateral surplus given $m$ will not improve their bilateral surplus in any other market structure. This is because a deviation on a contingency will not be observed by the other party on subsequent negotiations and will lead to the either the rejection equilibrium 
posited earlier or no agreement in that negotiation. Given feasibility, in either case, a deviation will reduce bilateral surplus. Hence, $U_{A}$ and $D_{1}$ will not deviate from the conjectured equilibrium. Applying this logic to all four negotiations demonstrates that the equilibrium outcomes in Propositions 1,2 and 4 are also equilibrium outcomes where pairs can negotiate binding breakdown contingent contracts.

Proof of Proposition 3

Suppose that $\pi_{i}\left(q_{i A}, q_{i B}, q_{-i A}, q_{-i B}\right)=P\left(q_{1 A}+q_{1 B}, q_{2 A}+q_{2 B}\right)\left(q_{i A}+q_{i B}\right)$. Then, under both upstream monopoly and competition, with non-integration, equilibrium quantities are determined by:

$$
\begin{aligned}
& q_{1 A}: \frac{\partial P}{\partial q_{1 A}}\left(q_{1 A}+q_{1 B}\right)+P(.) \leq \frac{\partial c_{A}}{\partial q_{A}} \\
& q_{1 B}: \frac{\partial P}{\partial q_{1 B}}\left(q_{1 A}+q_{1 B}\right)+P(.) \leq \frac{\partial c_{B}}{\partial q_{1 B}} \\
& q_{2 A}: \frac{\partial P}{\partial q_{2 A}}\left(q_{2 A}+q_{2 B}\right)+P(.) \leq \frac{\partial c_{A}}{\partial q_{2 A}} \\
& q_{2 B}: \frac{\partial P}{\partial q_{2 B}}\left(q_{2 A}+q_{2 B}\right)+P(.) \leq \frac{\partial c_{B}}{\partial q_{2 B}}
\end{aligned}
$$

Suppose that each downstream firm was supplied positive input quantities from each upstream firm and each of the above conditions held with equality. Then, $q_{1 A}+q_{1 B}$ must equal $q_{2 A}+q_{2 B}$. Note that if, say, both $q_{1 A}$ and $q_{1 B}$ are strictly positive, both (16) and (17) hold with equality implying that $\frac{\partial c_{A}}{\partial q_{1 A}}=\frac{\partial c_{A}}{\partial q_{2 A}}$. This can only be true if isoquants are linear (in which case any combination of $q_{1 A}$ and $q_{1 B}$ satisfying $q_{1 A}+q_{1 B}$ is an equilibrium. If isoquants are strictly concave, then $\frac{\partial c_{A}}{\partial q_{1 A}} \neq \frac{\partial c_{A}}{\partial q_{2 A}}$ implying that either one of (16) and (17) hold with equality with the other being a strict inequality. Applying the same logic to $D_{2}$ 's inputs, an equilibrium outcome exists that involves $q_{2 A}=q_{1 B}=0$ and $q_{1 A}=q_{2 B}$ at their Cournot equilibrium quantities with (16) and (19) holding with equality but (17) and (18) have a strict inequality if isoquants are strictly concave (as $\frac{\partial c_{A}}{\partial q_{1 A}}<\frac{\partial c_{A}}{\partial q_{2 A}}$ and $\frac{\partial c_{B}}{\partial q_{1 B}}<\frac{\partial c_{B}}{\partial q_{2 B}}$ ) and having an equality if isoquants are linear (as $\frac{\partial c_{A}}{\partial q_{1 A}}=\frac{\partial c_{A}}{\partial q_{2 A}}$ and $\left.\frac{\partial c_{B}}{\partial q_{1 B}}=\frac{\partial c_{B}}{\partial q_{2 B}}\right)$.

Under upstream monopoly (ii), with vertical integration, equilibrium quantities are determined by:

$$
\begin{gathered}
q_{1 A}: \frac{\partial P}{\partial q_{1 A}}\left(q_{1 A}+q_{1 B}\right)+P(.) \leq \frac{\partial c_{A}}{\partial q_{1 A}} \\
q_{1 B}: \frac{\partial P}{\partial q_{1 B}}\left(q_{1 A}+q_{1 B}\right)+P(.) \leq \frac{\partial c_{B}}{\partial q_{1 B}} \\
q_{2 A}: \frac{\partial P}{\partial q_{2 A}}\left(q_{1 A}+q_{1 B}+q_{2 A}+q_{2 B}\right)+P(.) \leq \frac{\partial c_{A}}{\partial q_{2 A}} \\
q_{2 B}: \frac{\partial P}{\partial q_{2 B}}\left(q_{1 A}+q_{1 B}+q_{2 A}+q_{2 B}\right)+P(.) \leq \frac{\partial c_{B}}{\partial q_{2 B}}
\end{gathered}
$$

If (20) and (21) hold with equality, because $\frac{\partial P}{\partial q_{1 j}}<0$, (22) and (23) are only satisfied if $q_{2 A}+q_{2 B}=0$ while (20) and (21) cannot hold if $q_{2 A}+q_{2 B}>0$ and (22) and (23) hold. As 
$q_{2 A}+q_{2 B}=0$, given the perfect substitutes assumption, industry profits are maximized under upstream monopoly. Moreover when $q_{1 B}+q_{2 B}=0$, the only way (20) and (22) can simultaneously hold is if $q_{2 A}=0$. Hence, $\hat{\hat{\Pi}}\left(\overline{D_{1} D_{2} U_{A} U_{B}}\right)=\Pi\left(\overline{D_{1} D_{2} U_{A} U_{B}}\right)$. The case for $\hat{\hat{\Pi}}\left(\overline{D_{1} D_{2} U_{A}}\right)=\Pi\left(\overline{D_{1} D_{2} U_{A}}\right)$ follows analogously.

Under upstream competition (i), (23) is still as in (19) In this case, the only way all four inequalities can be satisfied is if $q_{1 B}=q_{2 A}=0$; in which case, given the homogeneity of upstream costs, equilibrium downstream outputs are at their Cournot levels and so total industry profits remains the same as under non-integration. Note that the perfect substitutes assumption is not required in this case.

\section{Appendix B: Feasibility Conditions}

In this appendix, we provide explicitly, the conditions for our solution in Table 2 to be feasible. However, we do this for the special case where both upstream and both downstream firms are symmetric. While this simplifies notation, it is not an innocuous assumption. Indeed, it is precisely where one firm is far more productive than another that feasibility may breakdown. Thus, our purpose here is to give a feel for the conditions rather than a comprehensive treatment.

For the symmetric case, under non-integration, feasibility requires the following three inequalities be satisfied:

$\hat{\Pi}\left(D_{1} D_{2} U_{j}\right) \geq \frac{1}{2} \Pi\left(D_{i} U_{j}\right)$

$$
\left.\begin{array}{l}
\hat{\Pi}_{-1}\left(D_{1} D_{2} U_{A} U_{B}\right) \geq \max \left\{\begin{array}{l}
\hat{\Pi}\left(D_{1} D_{2} U_{i}\right)-\frac{1}{3}\left(\Pi\left(D_{i} U_{A} U_{B}\right)+\Pi\left(D_{i} U_{j}\right)\right), \\
\Pi\left(D_{i} U_{A} U_{B}\right)-\frac{1}{3}\left(\hat{\Pi}\left(D_{1} D_{2} U_{i}\right)+\Pi\left(D_{i} U_{j}\right)\right), \\
2 \Pi\left(D_{i} U_{j}, D_{-i} U_{-j}\right)-\frac{1}{3}\left(\hat{\Pi}\left(D_{1} D_{2} U_{i}\right)+\Pi\left(D_{i} U_{A} U_{B}\right)-\Pi\left(D_{i} U_{j}\right)\right)
\end{array}\right\} \\
\hat{\Pi}\left(D_{1} D_{2} U_{A} U_{B}\right)+\frac{1}{3}\left(\hat{\Pi}\left(D_{1} D_{2} U_{i}\right)+\Pi\left(D_{i} U_{A} U_{B}\right)-\Pi\left(D_{i} U_{j}\right)\right) \geq \hat{\Pi}_{-1}\left(D_{1} D_{2} U_{A} U_{B}\right)
\end{array}\right\}
$$

where $\hat{\Pi}_{-1}$ is equilibrium industry profits where one downstream-upstream pair canno trade with one another. Natural sufficient conditions for these to be satisfied are that $\hat{\Pi}\left(D_{1} D_{2} U_{A} U_{B}\right) \geq \hat{\Pi}_{-1}\left(D_{1} D_{2} U_{A} U_{B}\right) \geq \max \left\{\hat{\Pi}\left(D_{1} D_{2} U_{j}\right), \Pi\left(D_{i} U_{A} U_{B}\right), 2 \Pi\left(D_{i} U_{j}, D_{-i} U_{-j}\right)\right\}$.

Notice that these collapse to $\hat{\Pi}\left(D_{1} D_{2} U_{j}\right) \geq \frac{1}{2} \Pi\left(D_{i} U_{j}\right)$ if $D_{1}$ and $D_{2}$ produce final goods that are perfect substitutes and $U_{A}$ and $U_{B}$ are perfectly substitutable for one another (say having identical constant cost functions)

$\hat{\Pi}\left(D_{1} D_{2} U_{A} U_{B}\right)=\hat{\Pi}_{-1}\left(D_{1} D_{2} U_{A} U_{B}\right)=\hat{\Pi}\left(D_{1} D_{2} U_{i}\right)=2 \Pi\left(D_{i} U_{j}, D_{-i} U_{-j}\right)$ condition is equivalent to: $\frac{7}{72}(1-c)^{2}>0$. Thus, the feasibility condition is satisfied. 
When integration occurs, the feasibility conditions will be contingent upon whether there is forward or backwards integration. In this case, however, we can gather further information from the fact that integration is possible; namely that the acquired firm still operates and hence, their payoffs must be feasible. So if $U_{A}$ took over $D_{1}$, then an agreement with $D_{1}$ will be presumed to be feasible. This means that it must be the case that: $\hat{\hat{\Pi}}\left(D_{1} D_{2} U_{A} U_{B}\right)+\hat{\hat{\Pi}}\left(D_{1} D_{2} U_{i}\right) \geq 2 \Pi\left(D_{i} U_{A} U_{B}\right)$. Under symmetry, in addition to conditions under non-integration, the following conditions are required for feasibility:

$$
3 \hat{\Pi}_{-B 2}\left(D_{1} D_{2} U_{A} U_{B}\right)+\hat{\hat{\Pi}}\left(D_{1} D_{2} U_{i}\right)-3 \Pi\left(D_{i} U_{A} U_{B}\right)+\Pi\left(D_{i} U_{j}\right) \geq 0
$$

$3 \hat{\Pi}_{-B 1}\left(D_{1} D_{2} U_{A} U_{B}\right)+\Pi\left(D_{i} U_{j}\right) \geq \max \left\{3 \Pi\left(D_{i} U_{A} U_{B}\right)-\hat{\hat{\Pi}}\left(D_{1} D_{2} U_{i}\right), 3 \hat{\hat{\Pi}}\left(D_{1} D_{2} U_{i}\right)-\Pi\left(D_{i} U_{A} U_{B}\right)\right\}$

$$
\hat{\hat{\Pi}}_{-A 2}\left(D_{1} D_{2} U_{A} U_{B}\right)-\hat{\hat{\Pi}}\left(D_{1} D_{2} U_{i}\right)+\Pi\left(D_{i} U_{j}\right) \geq 0
$$

$$
3 \hat{\hat{\Pi}}\left(D_{1} D_{2} U_{A} U_{B}\right)+\Pi\left(D_{1} U_{A} U_{B}\right)-\Pi\left(D_{i} U_{j}\right) \geq \max \left\{\begin{array}{l}
3 \hat{\hat{\Pi}}_{-B 1}\left(D_{1} D_{2} U_{A} U_{B}\right), \\
3 \hat{\hat{\Pi}}_{-A 2}\left(D_{1} D_{2} U_{A} U_{B}\right)-\hat{\hat{\Pi}}\left(D_{1} D_{2} U_{i}\right), \\
3 \hat{\hat{\Pi}}_{-B 2}\left(D_{1} D_{2} U_{A} U_{B}\right)-2 \Pi\left(D_{i} U_{j}\right)
\end{array}\right\}
$$

For the case the perfect substitutes upstream and downstream, these reduce to: $\hat{\Pi}\left(D_{1} D_{2} U_{i}\right) \geq \frac{1}{3} \Pi\left(D_{i} U_{j}\right)$ (a weaker condition than that for non-integration).

\section{Appendix C: Merger Game}

Here we analyse the merger game when upstream and downstream firms are not necessarily symmetric but $U_{A}$ and $D_{1}$ have a stand-alone incentive to merge. We focus here on the BI case; the analysis of FI is similar. Let,

$$
\begin{gathered}
S_{A 1} \equiv \frac{1}{2}\left(\hat{\hat{\Pi}}_{A 1}\left(\overline{D_{1} D_{2} U_{A} U_{B}}\right)-\hat{\Pi}_{N I}\left(\overline{D_{1} D_{2} U_{A} U_{B}}\right)\right)+\frac{1}{6} \omega_{1} \\
S_{B 2} \equiv \frac{1}{2}\left(\hat{\hat{\Pi}}_{B 2}\left(\overline{D_{1} D_{2} U_{A} U_{B}}\right)-\hat{\Pi}_{N I}\left(\overline{D_{1} D_{2} U_{A} U_{B}}\right)\right)+\frac{1}{6} \omega_{2} \\
F_{A 1} \equiv \frac{1}{2}\left(\hat{\hat{\Pi}}_{C I}\left(\overline{D_{1} D_{2} U_{A} U_{B}}\right)-\hat{\hat{\Pi}}_{B 2}\left(\overline{D_{1} D_{2} U_{A} U_{B}}\right)\right)+\frac{1}{6} \omega_{1} \\
F_{B 2} \equiv \frac{1}{2}\left(\hat{\hat{\Pi}}_{C I}\left(\overline{D_{1} D_{2} U_{A} U_{B}}\right)-\hat{\hat{\Pi}}_{A 1}\left(\overline{D_{1} D_{2} U_{A} U_{B}}\right)\right)+\frac{1}{6} \omega_{2} \\
C_{A 1} \equiv \frac{1}{2}\left(\hat{\hat{\Pi}}_{C I}\left(\overline{D_{1} D_{2} U_{A} U_{B}}\right)-\hat{\Pi}_{N I}\left(\overline{D_{1} D_{2} U_{A} U_{B}}\right)\right)+\frac{1}{6}\left(\omega_{1}-\omega_{2}\right)
\end{gathered}
$$




$$
C_{B 2} \equiv \frac{1}{2}\left(\hat{\hat{\Pi}}_{C I}\left(\overline{D_{1} D_{2} U_{A} U_{B}}\right)-\hat{\Pi}_{N I}\left(\overline{D_{1} D_{2} U_{A} U_{B}}\right)\right)+\frac{1}{6}\left(\omega_{2}-\omega_{1}\right)
$$

where $\omega_{1}=\hat{\hat{\Pi}}_{A 1}\left(\overline{D_{1} D_{2} U_{A}}\right)-\hat{\Pi}_{N I}\left(\overline{D_{1} D_{2} U_{A}}\right)+\Pi\left(\overline{D_{2} U_{A} U_{B}}\right)-\Pi\left(\overline{D_{2} U_{B}}\right)$, $\omega_{2}=\hat{\Pi}_{B 2}\left(\overline{D_{1} D_{2} U_{B}}\right)-\hat{\Pi}_{N I}\left(\overline{D_{1} D_{2} U_{B}}\right)+\Pi\left(\overline{D_{1} U_{A} U_{B}}\right)-\Pi\left(\overline{D_{1} U_{A}}\right)$.

Here $S_{x}$ is the stand-alone merger incentive for pair $x, F_{x}$ is its follow-on incentive and $C_{x}$ is the pair's preference for both mergers as opposed to non-integration. Recall that we require the incentive to merge to be strictly positive in order for the merger to proceed and we assume that $S_{A 1}>0$.

We are interested here in the conditions that lead to (pure strategy) equilibria with complete integration $(\mathrm{CI})$, no integration $(\mathrm{NI})$ and partial integration (PI). A critical condition driving the existence of one type of equilibrium or the other is whether there is a follow-on incentive for a counter-merger. In this regard there are four broad cases.

Case 1: $F_{B 2}, F_{A 1} \leq 0$. In this case, both pairs prefer PI where the other pair merges to CI. As $S_{A 1}>0$, this means that PI where $U_{A}$ and $D_{1}$ are the only merging pair is always a subgame perfect equilibrium. If, in addition, $S_{B 2}>0$, there is another PI equilibrium with a sole merger between $U_{B}$ and $D_{2}$.

Case 2: $F_{B 2} \leq 0<F_{A 1}$. The PI equilibrium involving $U_{A}$ and $D_{1}$ continues to be an equilibrium in this case but the PI equilibrium involving $U_{B}$ and $D_{2}$ no longer exists. The only other candidate equilibrium is $\mathrm{CI}$; which exists if $C_{B 2}>0$. In this equilibrium, both pairs merge initially. $U_{A}-D_{1}$ deviating a merging second offers them no additional payoff. A $U_{B}-D_{2}$ deviation will result in NI as they do not engage in a follow-on merger; which strictly reduces their joint payoff.

Case 3: $F_{A 1} \leq 0<F_{B 2}$. The PI equilibrium involving $U_{A}$ and $D_{1}$ no longer exists. If $C_{A 1}>0$, a CI equilibrium exists as it is a dominant strategy for $U_{A}-D_{1}$ to merge initially. Given this, $U_{B}-D_{2}$ will counter-merge and are indifferent between doing this and merging initially. If $S_{B 2}>0$, a PI equilibrium involving $U_{B}$ and $D_{2}$ exists and an NI equilibrium does not exist. On the other hand, if $S_{B 2} \leq 0$, an NI equilibrium exists if $C_{A 1} \leq 0$ (as $U_{A^{-}}$ $D_{1}$ anticipates a counter-merger and prefers NI to $\mathrm{CI}$ ).

Case 4: $0<F_{A 1}, F_{B 2}$. No PI equilibrium exists. There is always a CI equilibrium where both pairs choose to merge initially and, given that the other firm is doing this, neither can, by not merging initially, commit not to counter-merge. Hence, they are indifferent between merging initially or not, so it is an equilibrium. If both $C_{A 1}, C_{B 2} \leq 0$, an NI equilibrium also exists. In this equilibrium, both pairs choose not to merge initially and neither deviates as they know this will lead to a counter-merger and CI (something which 
reduces their payoff).

In summary, the conditions that support the four possible equilibrium types are:

- NI: (1) $F_{A 1} \leq 0<F_{B 2}, S_{B 2} \leq 0$ and $C_{A 1} \leq 0$; (2) $0<F_{A 1}, F_{B 2}$ and $C_{A 1}, C_{B 2} \leq 0$.

- PI (involving $\left.U_{A}-D_{1}\right): F_{B 2} \leq 0$;

- PI (involving $U_{B}-D_{2}$ ): $F_{A 1} \leq 0$ and $S_{B 2}>0$;

- CI: (1) $0<F_{A 1}$ and $C_{B 2}>0$; (2) $0<F_{B 2}$ and $C_{A 1}>0$; (3) $0<F_{A 1}, F_{B 2}$.

There are three interesting specific cases to consider. First, under the conditions of Proposition 3, both $\omega_{1}$ and $\omega_{2}$ are positive. This rules out PI as an equilibrium as, under those conditions, industry profits are the same regardless of whether there is NI, PI or CI. For the same reason it rules out the first type of NI equilibrium (but for a special case) as both $S_{A 1}$ and $S_{B 2}$ must be non-negative. It does not, however, rule out the second type of NI equilibrium (again except for a special case) as either $C_{A 1}$ and $C_{B 2}$ must be nonnegative and this is certainly possible. Thus, both NI and CI remain equilibrium possibilities.

Second, suppose there are no externalities. In this case, PI involving $U_{A}$ and $D_{1}$ merging requires that $\Pi\left(\overline{D_{2} U_{A} U_{B}}\right)>\Pi\left(\overline{D_{2} U_{B}}\right)$ and $\Pi\left(\overline{D_{1} U_{A} U_{B}}\right)=\Pi\left(\overline{D_{1} U_{A}}\right)$. However, if $U_{B}$ and $D_{2}$ face additional merger costs, this condition can be relaxed. Nonetheless, both NI and CI remain possible equilibrium outcomes (for the same reasons as in the previous paragraph).

Finally, suppose upstream and downstream firms are symmetric. Then PI is only an equilibrium if $F_{A 1}=F_{B 2} \leq 0$. If $0<F_{A 1}=F_{B 2}, \mathrm{CI}$ is also an equilibrium but $\mathrm{NI}$ is only an equilibrium if $\hat{\hat{\Pi}}_{C I}\left(\overline{D_{1} D_{2} U_{A} U_{B}}\right) \leq \hat{\Pi}_{N I}\left(\overline{D_{1} D_{2} U_{A} U_{B}}\right)$.

We now turn to consider the OSS merger game. It is very similar to that of HT but with a more asymmetric 'playing field.' Their game has two stages. In stage $1, D_{1}$ and $D_{2}$ bid to acquire $U_{A}$. If $U_{A}$ rejects both bids, there is NI. If $U_{A}$ accepts a bid, the losing downstream firm bids for $U_{B}$. If this accepted there is CI, otherwise there is PI. This game is depicted in Figure 9.

To simplify exposition, suppose that if any firm acquires $U_{A}$, it is $D_{1}$ (see Chen, 2001, for a discussion). Working backwards, if this happens, $D_{2}$ will acquire $U_{B}$ if and only if $F_{B 2}>0$. Thus, there are two cases to consider. First, if $F_{B 2} \leq 0$, PI occurs if $S_{A 1}>0$ and NI occurs otherwise. Second, if $F_{B 2}>0$, then CI occurs if $C_{A 1}>0$; otherwise, NI is the unique equilibrium. Therefore, in contrast to the HT game, there is always a unique subgame perfect equilibrium outcome. Other than that, incentives for an initial merger are driven by the same conditions as in the HT game. 
Figure 1: Extensive Form Game

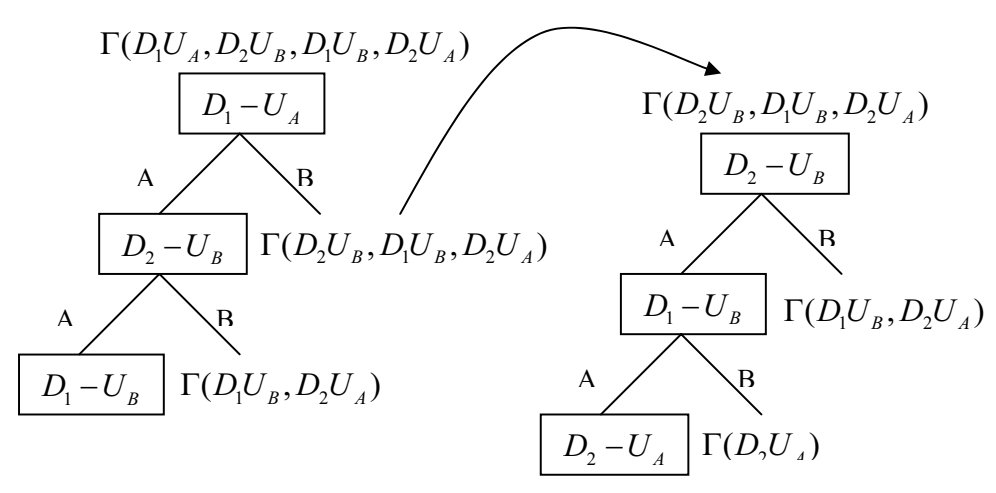


Figure 2: Upstream Competition

Patterns of Negotiation

(a) Non-Integration

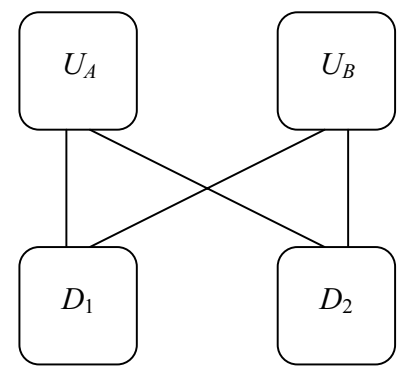

(b) Forward Integration

(c) Backwards Integration
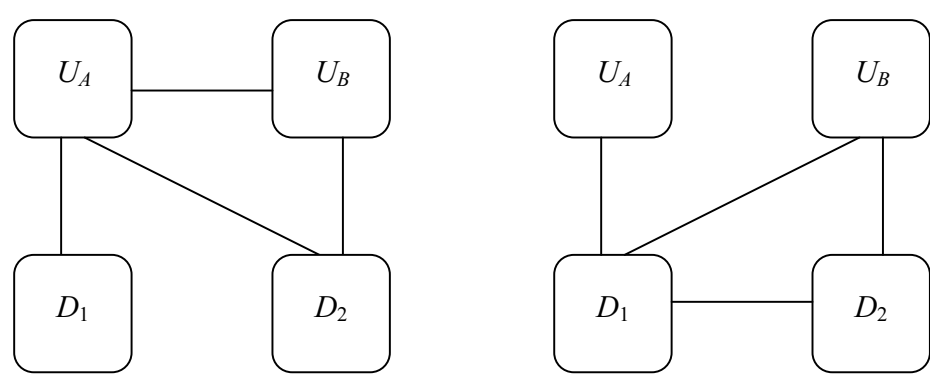
Figure 3: Upstream Monopoly

Patterns of Negotiation

(a) Non-Integration

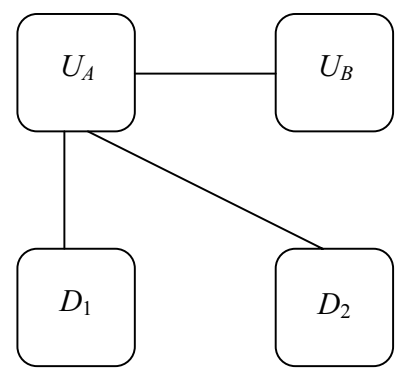

(b) Forward Integration

(c) Backwards Integration
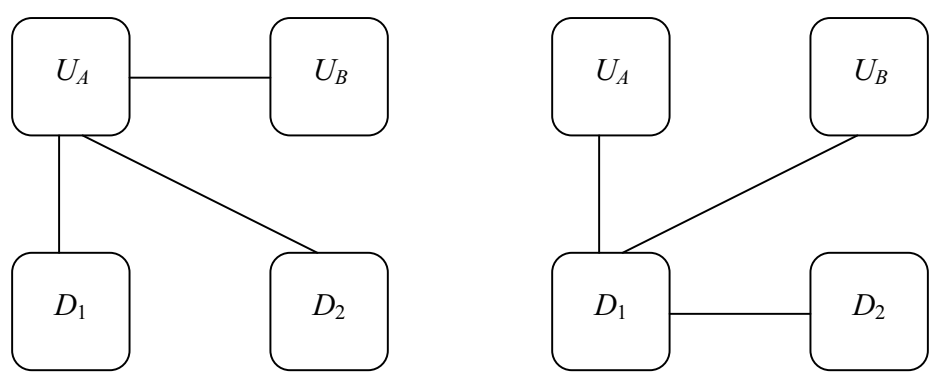
Figure 7: HT's Merger Game

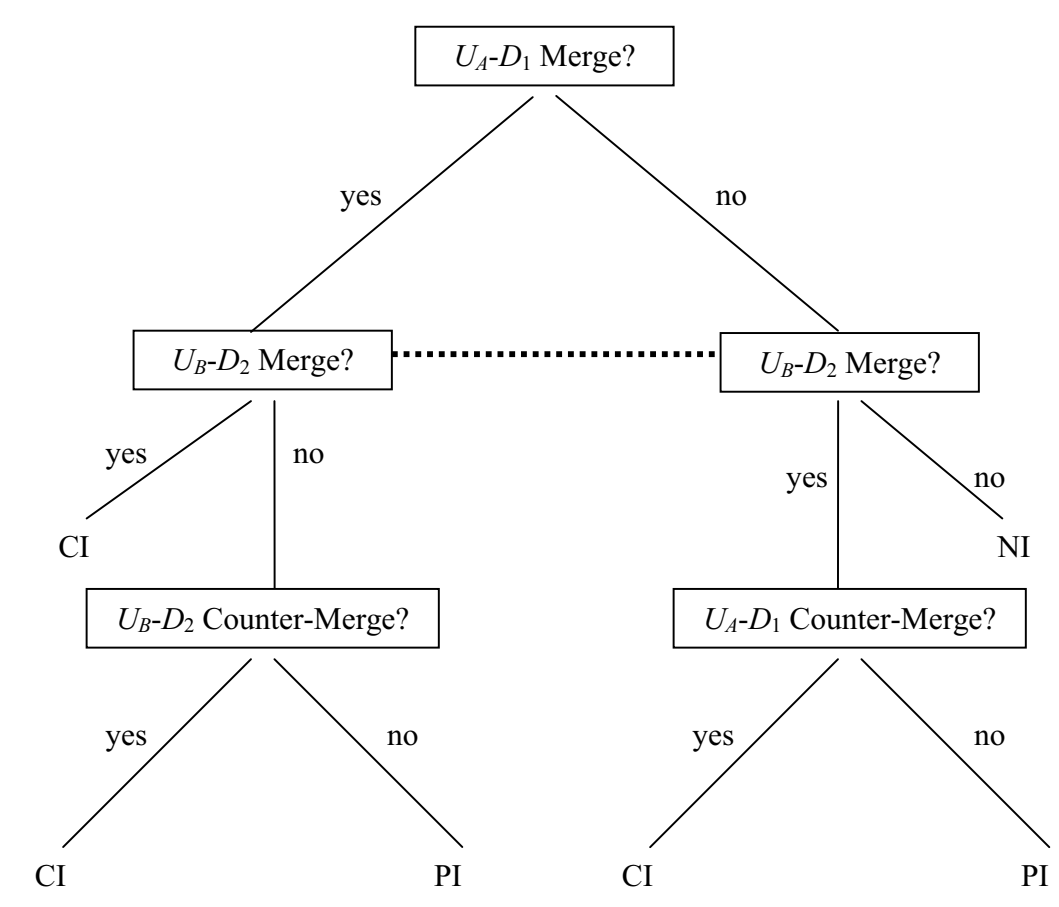


Figure 9: OSS's Merger Game

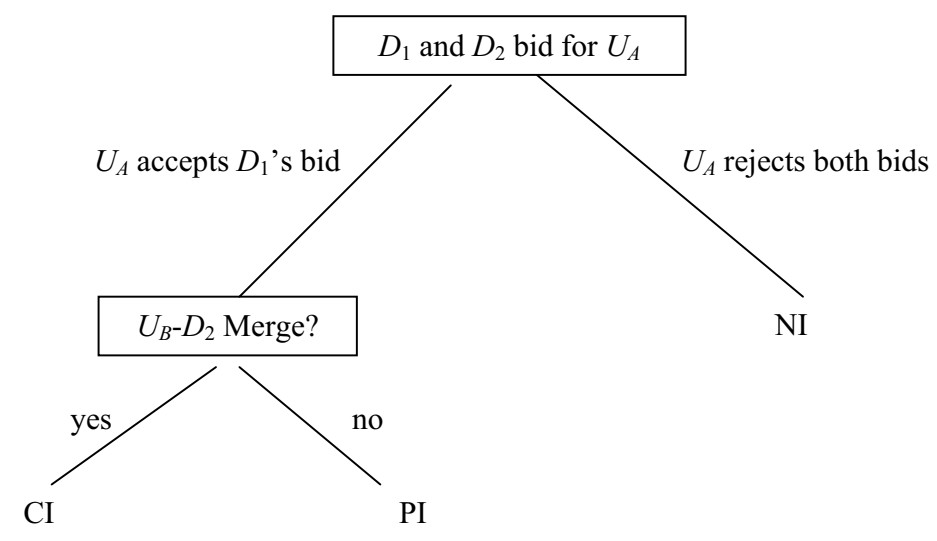


Table 1: Payoffs in No Externality Case

(where $(x, y)=(1,1)$ for NI, $(0,1)$ for FI and $(1,0)$ for BI)

\begin{tabular}{|c|c|}
\hline Upstream Competition & Upstream Monopoly $\left(U_{A}\right.$ owns $\left.U_{B}\right)$ \\
\hline$v_{D_{1}}=\frac{1}{12}\left(\begin{array}{l}3 \Pi\left(\overline{D_{1} D_{2} U_{A} U_{B}}\right)+\Pi\left(\overline{D_{1} D_{2} U_{A}}\right) \\
+\Pi\left(\overline{D_{1} U_{A} U_{B}}\right)+\Pi\left(\overline{D_{1} U_{A}}\right)-3 \Pi\left(\overline{D_{2} U_{B}}\right) \\
+x\left(\Pi\left(\overline{D_{1} D_{2} U_{B}}\right)-\Pi\left(\overline{D_{2} U_{B}}\right)+\Pi\left(\overline{D_{1} U_{B}}\right)\right) \\
+y\left(-3\left(\Pi\left(\overline{D_{2} U_{A} U_{B}}\right)-\Pi\left(\overline{D_{2} U_{B}}\right)-\Pi\left(\overline{D_{2} U_{A}}\right)\right)\right.\end{array}\right)$ & $v_{D_{1}}=\frac{1}{12}\left(\begin{array}{l}3 \Pi\left(\overline{D_{1} D_{2} U_{A} U_{B}}\right)+\Pi\left(\overline{D_{1} D_{2} U_{A}}\right) \\
+\Pi\left(\overline{D_{1} U_{A} U_{B}}\right)+\Pi\left(\overline{D_{1} U_{A}}\right) \\
+(1-y) \Pi\left(\overline{D_{1} D_{2} U_{B}}\right)-3 y \Pi\left(\overline{D_{2} U_{A} U_{B}}\right) \\
-y \Pi\left(\overline{D_{2} U_{A}}\right)+(1-y) \Pi\left(\overline{D_{1} U_{B}}\right)\end{array}\right)$ \\
\hline$v_{D_{2}}=\frac{1}{12}\left(\begin{array}{l}3 \Pi\left(\overline{D_{1} D_{2} U_{A} U_{B}}\right)+\Pi\left(\overline{D_{1} D_{2} U_{A}}\right) \\
-3 \Pi\left(\overline{D_{1} U_{A} U_{B}}\right)-\Pi\left(\overline{D_{1} U_{A}}\right)+3 \Pi\left(\overline{D_{2} U_{B}}\right) \\
+x\left(\Pi\left(\overline{D_{1} D_{2} U_{B}}\right)-\Pi\left(\overline{D_{2} U_{B}}\right)-\Pi\left(\overline{D_{1} U_{B}}\right)\right) \\
+y\left(\Pi\left(\overline{D_{2} U_{A} U_{B}}\right)-\Pi\left(\overline{D_{2} U_{B}}\right)+\Pi\left(\overline{D_{2} U_{A}}\right)\right)\end{array}\right)$ & $v_{D_{2}}=\frac{1}{12}\left(\begin{array}{l}3 \Pi\left(\overline{D_{1} D_{2} U_{A} U_{B}}\right)+\Pi\left(\overline{D_{1} D_{2} U_{A}}\right) \\
-3 \Pi\left(\overline{D_{1} U_{A} U_{B}}\right)-\Pi\left(\overline{D_{1} U_{A}}\right) \\
+(1-y) \Pi\left(\overline{D_{1} D_{2} U_{B}}\right)+y \Pi\left(\overline{D_{2} U_{A} U_{B}}\right) \\
+y \Pi\left(\overline{D_{2} U_{A}}\right)-(1-y) \Pi\left(\overline{D_{1} U_{B}}\right)\end{array}\right)$ \\
\hline$v_{U_{A}}=\frac{1}{12}\left(\begin{array}{l}3 \Pi\left(\overline{D_{1} D_{2} U_{A} U_{B}}\right)+\Pi\left(\overline{\left(\overline{D_{1} D_{2} U_{A}}\right.}\right) \\
+\Pi\left(\overline{D_{1} U_{A} U_{B}}\right)+\Pi\left(\overline{D_{1} U_{A}}\right)-3 \Pi\left(\overline{D_{2} U_{B}}\right) \\
+x\left(-3\left(\overline{\left(\Pi\left(\overline{D_{1} D_{2} U_{B}}\right)\right.}-\Pi\left(\overline{D_{2} U_{B}}\right)\right)-\Pi\left(\overline{D_{1} U_{B}}\right)\right) \\
+y\left(\Pi\left(\overline{D_{2} U_{A} U_{B}}\right)-\Pi\left(\overline{D_{2} U_{B}}\right)+\Pi\left(\overline{D_{2} U_{A}}\right)\right)\end{array}\right)$ & $v_{U_{A}}=\frac{1}{12}\left(\begin{array}{l}3 \Pi\left(\overline{D_{1} D_{2} U_{A} U_{B}}\right)+\Pi\left(\overline{D_{1} D_{2} U_{A}}\right) \\
+\Pi\left(\overline{D_{1} U_{A} U_{B}}\right)+\Pi\left(\overline{D_{1} U_{A}}\right) \\
-3(1-y) \Pi\left(\overline{D_{1} D_{2} U_{B}}\right)+y \Pi\left(\overline{D_{2} U_{A} U_{B}}\right) \\
+y \Pi\left(\overline{D_{2} U_{A}}\right)-(1-y) \Pi\left(\overline{D_{1} U_{B}}\right)\end{array}\right)$ \\
\hline$v_{U_{B}}=\frac{1}{12}\left(\begin{array}{l}3 \Pi\left(\overline{\bar{D}_{1} D_{2} U_{A} U_{B}}\right)-3 \Pi\left(\overline{D_{1} D_{2} U_{A}}\right) \\
+\Pi\left(\overline{D_{1} U_{A} U_{B}}\right)-\Pi\left(\overline{D_{1} U_{A}}\right)+3 \Pi\left(\overline{D_{2} U_{B}}\right) \\
+x\left(\Pi\left(\overline{D_{1} D_{2} U_{B}}\right)-\Pi\left(\overline{D_{2} U_{B}}\right)+\Pi\left(\overline{D_{1} U_{B}}\right)\right) \\
+y\left(\Pi\left(\overline{D_{2} U_{A} U_{B}}\right)-\Pi\left(\overline{D_{2} U_{B}}\right)-\Pi\left(\overline{D_{2} U_{A}}\right)\right)\end{array}\right)$ & $v_{U_{B}}=\frac{1}{12}\left(\begin{array}{l}3 \Pi\left(\overline{D_{1} D_{2} U_{A} U_{B}}\right)-3 \Pi\left(\overline{D_{1} D_{2} U_{A}}\right) \\
+\Pi\left(\overline{D_{1} U_{A} U_{B}}\right)-\Pi\left(\overline{D_{1} U_{A}}\right) \\
+(1-y) \Pi\left(\overline{D_{1} D_{2} U_{B}}\right)+y \Pi\left(\overline{D_{2} U_{A} U_{B}}\right) \\
-y \Pi\left(\overline{D_{2} U_{A}}\right)+(1-y) \Pi\left(\overline{D_{1} U_{B}}\right)\end{array}\right)$ \\
\hline$\frac{\partial\left(v_{D_{1}}+v_{U_{A}}\right)}{\partial(-x)}=\frac{1}{6}\left(\Pi\left(\overline{D_{1} D_{2} U_{B}}\right)-\Pi\left(\overline{D_{2} U_{B}}\right)\right)$ & $\frac{\partial\left(v_{D_{1}}+v_{U_{A}}\right)}{\partial(-x)}=0$ \\
\hline$\frac{\partial\left(v_{D_{1}}+v_{U_{A}}\right)}{\partial(-y)}=\frac{1}{6}\left(\Pi\left(\overline{D_{2} U_{A} U_{B}}\right)-\Pi\left(\overline{D_{2} U_{B}}\right)\right)$ & $\frac{\partial\left(v_{D_{1}}+v_{U_{A}}\right)}{\partial(-y)}=\frac{1}{6}$ \\
\hline
\end{tabular}


Table 2: Payoffs in Competitive Externality Case

$($ where $(x, y)=(1,1)$ for $\mathrm{NI},(0,1)$ for $\mathrm{FI}$ and $(1,0)$ for $\mathrm{BI})$

\begin{tabular}{|c|c|}
\hline Upstrea & Upstr \\
\hline 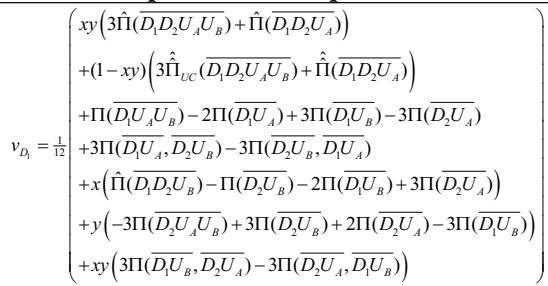 & $v_{D_{1}}=\frac{1}{12}\left(\begin{array}{l}x y\left(3 \hat{\Pi}\left(\overline{D_{1} D_{2} U_{A} U_{B}}\right)+\hat{\Pi}\left(\overline{D_{1} D_{2} U_{A}}\right)\right)+ \\
(1-x y)\left(3 \hat{\hat{\Pi}_{U M}}\left(\overline{D_{1} D_{2} U_{A} U_{B}}\right)+\hat{\hat{\Pi}}\left(\overline{D_{1} D_{2} U_{A}}\right)\right) \\
+(1-y) \hat{\Pi}\left(\overline{D_{1} D_{2} U_{B}}\right)-3 y \Pi\left(\overline{D_{1} U_{A}}\right) \\
-y \Pi\left(\overline{D_{2} U_{A}}\right)+(1-y) \Pi\left(\overline{D_{1} U_{B}}\right)\end{array}\right.$ \\
\hline 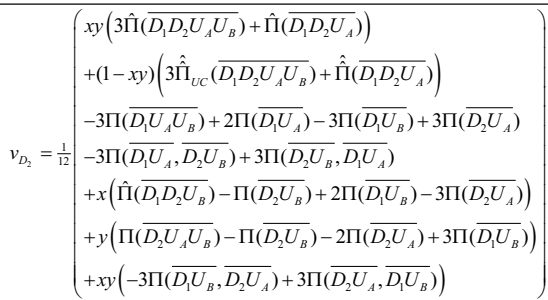 & $v_{D_{2}}=\frac{1}{12}\left(\begin{array}{l}x y\left(3 \hat{\Pi}\left(\overline{D_{1} D_{2} U_{A} U_{B}}\right)+\hat{\Pi}\left(\overline{D_{1} D_{2} U_{A}}\right)\right)+ \\
(1-x y)\left(3 \hat{\hat{\Pi}_{U M}}\left(\overline{D_{1} D_{2} U_{A} U_{B}}\right)+\hat{\hat{\Pi}}\left(\overline{D_{1} D_{2} U_{A}}\right)\right) \\
-3 \Pi\left(\overline{D_{1} U_{A} U_{B}}\right)-\Pi\left(\overline{D_{1} U_{A}}\right) \\
+(1-y) \hat{\Pi}\left(\overline{D_{1} D_{2} U_{B}}\right)+y \Pi\left(\overline{D_{2} U_{A} U_{B}}\right) \\
+y \Pi\left(\overline{D_{2} U_{A}}\right)-(1-y) \Pi\left(\overline{D_{1} U_{B}}\right)\end{array}\right.$ \\
\hline 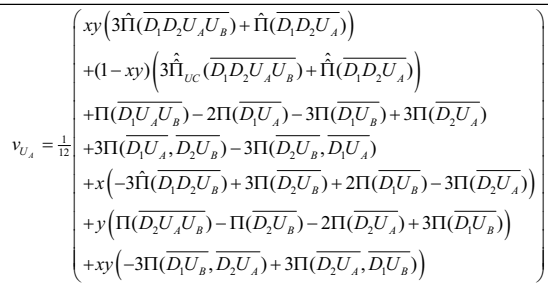 & $v_{U_{A}}=\frac{1}{12}\left(\begin{array}{l}x y\left(3 \hat{\Pi}\left(\overline{D_{1} D_{2} U_{A} U_{B}}\right)+\hat{\Pi}\left(\overline{D_{1} D_{2} U_{A}}\right)\right)+ \\
(1-x y)\left(3 \hat{\hat{\Pi}_{U M}}\left(\overline{D_{1} D_{2} U_{A} U_{B}}\right)+\hat{\hat{\Pi}}\left(\overline{D_{1} D_{A} U_{A}}\right)+\Pi\left(\overline{D_{1} U_{A}}\right)\right. \\
-3(1-y) \hat{\Pi}\left(\overline{D_{1} D_{2} U_{B}}\right)+y \Pi\left(\overline{D_{2} U_{A} U_{B}}\right) \\
+y \Pi\left(\overline{D_{2} U_{A}}\right)-(1-y) \Pi\left(\overline{D_{1} U_{B}}\right)\end{array}\right.$ \\
\hline 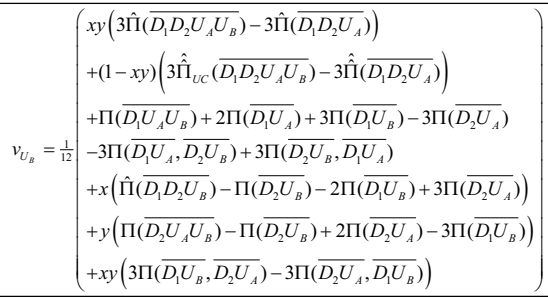 & $v_{U_{B}}=\frac{1}{12}\left(\begin{array}{l}x y\left(3 \hat{\Pi}\left(\overline{D_{1} D_{2} U_{A} U_{B}}\right)-3 \hat{\Pi}\left(\overline{D_{1} D_{2} U_{A}}\right)\right)+ \\
(1-x y)\left(3 \hat{\hat{\Pi}_{U M}}\left(\overline{D_{1} D_{2} U_{A} U_{B}}\right)-3 \hat{\Pi}\left(\overline{D_{1} D_{2} U_{A}}\right)\right) \\
+\Pi\left(\overline{D_{1} U_{A} U_{B}}\right)-\Pi\left(\overline{D_{1} U_{A}}\right) \\
+(1-y) \hat{\Pi}\left(\overline{D_{1} D_{2} U_{B}}\right)+y \Pi\left(\overline{D_{2} U_{A} U_{B}}\right) \\
-y \Pi\left(\overline{D_{2} U_{A}}\right)+(1-y) \Pi\left(\overline{D_{1} U_{B}}\right)\end{array}\right.$ \\
\hline $\begin{array}{r}\frac{\partial\left(v_{D_{1}}+v_{V_{A}}\right)}{\partial(-x)}=\frac{1}{2}\left(\hat{\hat{\Pi}}_{U C}\left(\overline{D_{1} D_{2} U_{A} U_{B}}\right)-\hat{\Pi}\left(\overline{D_{1} D_{2} U_{A} U_{B}}\right)\right) \\
+\frac{1}{6}\left(\begin{array}{l}\hat{\hat{\Pi}}\left(\overline{D_{1} D_{2} U_{A}}\right)-\hat{\Pi}\left(\overline{D_{1} D_{2} U_{A}}\right) \\
+\hat{\Pi}\left(\overline{D_{1} D_{2} U_{B}}\right)-\Pi\left(\overline{D_{2} U_{B}}\right)\end{array}\right)\end{array}$ & $\begin{array}{l}\left.\begin{array}{l}U M \\
\hat{D_{1} D_{2} U_{A} U_{B}}\end{array}\right)-\hat{\Pi}\left(\overline{D_{1} D_{2} U_{A} U_{B}}\right) \\
\left.\hat{\Pi}\left(\overline{D_{1} D_{2} U_{A}}\right)-\hat{\Pi}\left(\overline{D_{1} D_{2} U_{A}}\right)\right)\end{array}$ \\
\hline 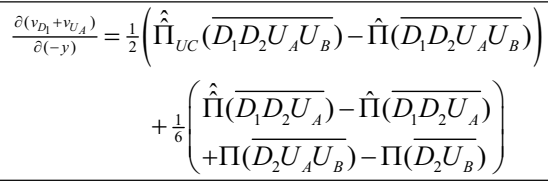 & $\begin{array}{l}\frac{1}{2}\left(\hat{\hat{\Pi}}_{U M}\left(\overline{D_{1} D_{2} U_{A} U_{B}}\right)-\hat{\Pi}\left(\overline{D_{1} D_{2} U_{A} U_{B}}\right)\right) \\
+\frac{1}{6}\left(\begin{array}{l}\hat{\hat{\Pi}}\left(\overline{D_{1} D_{2} U_{A}}\right)-\hat{\Pi}\left(\overline{D_{1} D_{2} U_{A}}\right) \\
+\Pi\left(\overline{D_{2} U_{A} U_{B}}\right)-\hat{\Pi}\left(\overline{D_{1} D_{2} U_{B}}\right)\end{array}\right)\end{array}$ \\
\hline
\end{tabular}


Table 3: Payoffs from Second Merger

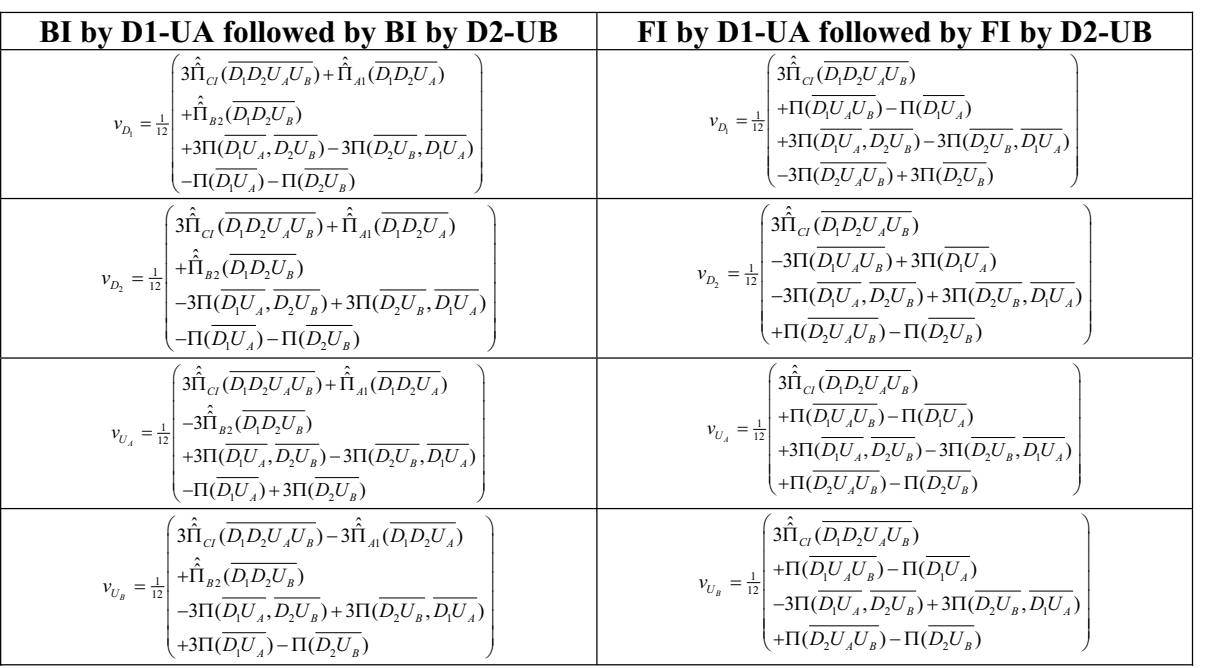




\section{References}

Aghion, P. and J. Tirole (1994), "The Management of Innovation," Quarterly Journal of Economics, 109 (4), pp.1185-1210.

Binmore, K., A. Rubinstein and A. Wolinsky (1986), "The Nash Bargaining Solution in Economic Modelling," RAND Journal of Economics, 17, 176-188.

Bjornerstedt, J. and J. Stennek (2001), "Bilateral Oligopoly," Working Paper, No.555, IUI, Stockholm.

Bolton, P. and M.D. Whinston (1993), "Incomplete Contracts, Vertical Integration, and Supply Constraints," Review of Economic Studies, 60 (1), pp.121-148.

Chandler, A.D. (1964), Strategy and Structure, MIT Press: Cambridge (MA).

Chemla, G. (2003), "Downstream Competition, Foreclosure and Vertical Integration," Journal of Economics and Management Strategy, 12 (2), pp.261-289.

Chen, Y. (2001), "On Vertical Mergers and their Competitive Effects," RAND Journal of Economics, 32 (4), pp.667-685.

Choi, J.P. and S.S. Yi (2000), "Vertical Foreclosure with the Choice of Inpu Specifications," RAND Journal of Economics, 31 (4), pp.717-743.

de Fontenay, C.C. and J.S. Gans (1999), "Extending Market Power Through Vertical Integration," Working Paper, Melbourne Business School.

de Fontenay, C.C. and J.S Gans (2003a), "Organizational Design and Technology Choice under Intrafirm Bargaining: Comment," American Economic Review, 93 (1), pp.448-455.

de Fontenay, C.C. and J.S. Gans (2003b), "Can Vertical Integration by a Monopsonis Harm Consumer Welfare?” Working Paper, No.2003-03, Melbourne Business School.

Dewatripont, M. (1989), "Renegotiation and Information Revelation over Time: The Case of Optimal Labor Contracts," Quarterly Journal of Economics, 104 (3), pp.589-619.

DOJ (1984), Non-Horizontal Merger Guidelines, www.usdoj.gov.

Gans, J.S. (2001), "Markets for Ownership,” Working Paper, Melbourne Business 
School.

Grossman, S. and O. Hart (1986), "The Costs and Benefits of Ownership: A Theory of Vertical and Lateral Integration,” Journal of Political Economy, 94, 691-719.

Hart, O. (1995), Firms, Contracts and Financial Structure, Oxford University Press: Oxford.

Hart, O. and J. Moore (1988), "Incomplete Contracts and Renegotiation," Econometrica, $56, \mathrm{pp} .755-786$

Hart, O. and J. Moore (1990), "Property Rights and the Theory of the Firm," Journal of Political Economy, 98 (6), pp.1119-1158.

Hart, O. and J. Tirole (1990), "Vertical Integration and Market Foreclosure," Brookings Papers on Economic Activity, Microeconomics, 205-285.

Hendricks, K. and R.P. McAfee (2000), "A Theory of Bilateral Oligopoly with Applications to Vertical Mergers," mimeo., Austin.

Hovenkamp, H. (2001), "Post-Chicago Antitrust: Review and Critique," Columbia Business Law Review, No.2:257, pp.257-337.

Inderst, R. and C. Wey (2003), "Bargaining, Mergers and Technology Choice in Bilaterally Oligopolistic Industries," RAND Journal of Economics, 34 (1), pp.119.

Jackson, M.O. and A. Wolinsky (1996), "A Strategic Model of Social and Economic Networks," Journal of Economic Theory, 71 (1), pp.44-74.

Klass, M.W. and M.A. Salinger (1995), "Do New Theories of Vertical Foreclosure Provide Sound Guidance for Consent Agreements in Vertical Merger Cases?" The Antitrust Bulletin, Fall, pp.667-698.

Klein, B., R. Crawford and A. Alchian (1978), "Vertical Integration, Appropriable Rents, and the Competitive Contracting Process," Journal of Law and Economics, 21 (2), pp.297-326.

Kranton, R.E. and D.F. Minehart (2001), "A Theory of Buyer-Seller Networks," American Economic Review, 91 (3), pp.485-508.

McAfee, R.P. and M. Schwartz (1994), "Opportunism in Multilateral Vertical Contracting: Nondiscrimination, Exclusivity and Uniformity," American Economic Review, 84 (1), 210-230.

McLaren, J. (2000), “'Globalization' and Vertical Structure," American Economic 
Review, 90 (5), pp.1239-1254.

Myerson, R. (1980), "Conference Structures and Fair Allocation Rules," International Journal of Game Theory, 9, pp.169-182.

O’Brien, D.P. and G. Shaffer (1992), "Vertical Control with Bilateral Contracts," RAND Journal of Economics, 23 (3), 299-308.

Ordover, J., G. Saloner and S. Salop (1990), "Equilibrium Vertical Foreclosure," American Economic Review, 80 (1), 127-142.

Rey, P. and J. Tirole (2003), “A Primer on Foreclosure," Handbook of Industrial Organization, Vol.III, North Holland: Amsterdam (forthcoming).

Rey, P. and T. Verge (2002), "Bilateral Control with Vertical Contracts," mimeo., Toulouse.

Riordan, M.H. and S.C. Salop (1995), "Evaluating Vertical Mergers: A Post-Chicago Approach," Antitrust Law Journal, 68, pp.513-568.

Scherer, F.M. and D. Ross (1990), Industrial Market Structure and Economic Performance, $3^{\text {rd }}$ Edition, Houghton-Mifflin: Boston.

Segal, I. (1999), “Contracting with Externalities," Quarterly Journal of Economics, 114 (2), pp.337-388.

Segal, I. and M. Whinston (2000), "Exclusive Contracts and Protection of Investments," RAND Journal of Economics, 31 (4), pp.603-633.

Segal, I. and M. Whinston (2003), "Robust Predictions for Bilateral Contracting with Externalities," Econometrica, 71 (3), pp.757-792.

Stole, L. and J. Zwiebel (1996), "Intra-firm Bargaining under Non-binding Contracts," Review of Economic Studies, 63 (3), 375-410.

Stole, L. and J. Zwiebel (1998), "Mergers, Employee Hold-Up and the Scope of the Firm: An Intrafirm Bargaining Approach to Mergers," mimeo., Stanford.

Williamson, O.E. (1975), Markets and Hierarchies: Analysis and Antitrust Implications, Free Press: New York.

Williamson, O.E. (1985), The Economic Institutions of Capitalism, Free Press: New York.

Williamson, O.E. (1987), "Antitrust Policy," in J. Eatwell, M. Milgate and P. Newman (eds.), The New Palgrave: A Dictionary of Economics, Vol.1, Macmillan: London, pp.95-97. 